\title{
Integrating polarized light over a planetary disk applied to starlight reflected by extrasolar planets
}

\author{
D. M. Stam ${ }^{1, \star}$, W. A. De Rooij ${ }^{2, \star \star}$, G. Cornet $^{2, \star}$, and J. W. Hovenier ${ }^{1}$ \\ 1 Astronomical Institute "Anton Pannekoek", University of Amsterdam, Kruislaan 403, 1098 SJ Amsterdam, The Netherlands \\ e-mail: D.M.Stam@sron.nl \\ 2 Department of Physics and Astronomy, Vrije Universiteit, De Boelelaan 1081, 1081 HV Amsterdam, The Netherlands
}

Received 17 October 2005 / Accepted 19 January 2006

ABSTRACT

\begin{abstract}
We present an efficient numerical method for integrating planetary radiation over a planetary disk, which is especially interesting for simulating signals of extrasolar planets. Our integration method is applicable to calculating the full flux vector of the disk-integrated planetary radiation, i.e. not only its observed flux (irradiance), but also its state of polarization (linear and circular). Including polarization is important for simulations of the light reflected by a planet, in particular, because this will generally be polarized. Our integration method is based on the expansion of the radiation field of a spherical, horizontally homogeneous planet into generalized spherical functions. With the expansion coefficients, the flux vector of the disk-integrated, reflected starlight can be obtained rapidly for arbitrary planetary phase angles. We describe the theory behind the disk-integration algorithm and results of accuracy tests. In addition, we give some illustrative examples of the application of the algorithm to extrasolar planets.
\end{abstract}

Key words. methods: numerical - polarization - radiative transfer - stars: planetary systems

\section{Introduction}

During the past decades, the spatial resolution of the observations of planets in our solar system has increased significantly. This increase stems from planetary missions such as Voyager, Galileo, and Cassini-Huygens, from space-bound telescopes like the Hubble Space Telescope, and from the development of ground-based adaptive optics systems. Together with this increasing spatial resolution, the spatial resolution of numerical simulations for the interpretation of observations of solar system planets has increased, too. Consequently, efficient numerical methods to integrate reflected starlight across a planetary disk have received little attention lately. Recent discoveries of extrasolar planets, however, have renewed interest in such numerical integration methods.

Because extrasolar planets are very faint compared to their parent star, and because the angular distance between a star and an orbiting planet is very small, observing the planet itself by detecting the stellar light it reflects or the thermal radiation it emits is extremely difficult. Consequently, almost all of the known extrasolar planets have been found by indirect methods, in which not the planet itself but rather its influence on its parent star is detected. Although very useful for detecting an extrasolar planet, indirect detection methods give, however, little information on the planet itself, apart from its mass and some orbital elements. Information on the physical structure and chemical composition of a planet, for example, can be derived from direct observations of the planetary radiation. To succeed in detecting the very faint planetary radiation, dedicated instruments and space missions

* Present address: SRON Netherlands Institute for Space Research, Sorbonnelaan 2, 3584 CA Utrecht, The Netherlands.

$\star \star$ Present address: Statistics Netherlands (CBS), Prinses Beatrixlaan 428, 2273 XZ Voorburg, The Netherlands. are being designed, such as the Planetfinder instrument that has been designed for use on one of ESO's VLTs and ESA's Darwin mission (Fridlund 2004) with space telescopes flying in formation and performing infrared interferometry. With Planetfinder, the aim is not only to detect giant, gaseous extrasolar planets but also to characterize them, i.e. to derive the chemical composition, sizes, and spatial distributions of the atmospheric constituents, such as haze and/or cloud particles. The Darwin mission will concentrate on terrestrial extrasolar planets.

Recently, the first direct observations of extrasolar planets have been reported (Chauvin et al. 2004, 2005; Charbonneau et al. 2005; Deming et al. 2005). Chauvin et al. (2004) present infrared adaptive-optics images and spectra showing water vapor in the atmosphere of a giant planet candidate around a brown dwarf. The observations of Charbonneau et al. (2005) and Deming et al. (2005) concern the infrared, thermal radiation of close-in extrasolar planets. The thermal signal of the planet could be deduced from the combined signal of the star and the planet by subtracting the stellar signal that was obtained at the time the planet was occulted by its star.

Direct observations of extrasolar planets, such as the images and spectra measured by Chauvin et al. (2004), will pertain to disk-integrated planetary radiation for years to come. For the numerical simulations that are used to (1) interpret such observations in terms of the physical structure and chemical composition of an extrasolar planet, and (2) fine-tune instruments for direct detection, it is therefore essential to accurately integrate planetary radiation over the planetary disk. Planetary radiation consists of thermally emitted radiation and reflected starlight. Thermal radiation that is emitted by the planet should usually be integrated over the planetary disk as a whole, whereas the integration of reflected starlight can be limited to the region of the disk that is illuminated by the star. Obviously, this illuminated 
region varies with the planetary phase angle, i.e. the angle between the star and the observer as seen from the center of the planet. Another difference between integrating thermal radiation and reflected starlight is that thermal radiation will usually be unpolarized, whereas reflected starlight will usually be polarized because it has been scattered by particles in the planetary atmosphere and/or it has been reflected by the planetary surface, if present. In this article, we concentrate on the integration of the reflected light over the planetary disk.

The disk-integration method we describe is based on the expansion method proposed by van de Hulst (1980), except that ours is an extended version that is applicable to integrating fullintensity vectors, thus not only the intensity but also the state of polarization (linear and circular) of the planetary radiation over the disk. Including the state of polarization of the light when integrating radiation over a planetary disk significantly complicates the problem, because in general both the degree and the direction of polarization varies across the disk. The diskintegration algorithm was first described in de Rooij (1985), and has been used in a study of the phase function and polarization of Titan (Stammes 1992), but has never been made available to the general public before. Since the discovery of extrasolar planets, the recent and (most certainly) future direct detections of these planets have renewed the interest in and need for efficient disk-integration methods. In this paper, we describe both the theory behind the disk-integration method, and a 5-step numerical recipe for its use. In addition, we offer some illustrative examples of the application of the algorithm to extrasolar planets.

Although complicated, including polarization in the diskintegration method is worthwhile, because it enables the numerical simulation of polarization observations of extrasolar planets, such as those planned with an instrument like Planetfinder (Feldt et al. 2003) on the VLT. Because polarimetry is a strong tool both for the detection and the characterization of extrasolar planets (see Seager et al. 2000; Stam 2003; Hough \& Lucas 2003; Saar \& Seager 2003; Stam et al. 2004), increased interest in the polarization of extrasolar planets is to be expected. Even without explicit polarization observations, using a vector representation of the reflected starlight instead of a scalar approximation is important in order to obtain accurate results when calculating fluxes (Sromovsky 2005; Stam \& Hovenier 2005), and when interpreting flux observations that have been performed with a polarization-sensitive instrument (Stam et al. 2000). In this article, we concentrate on describing the method for integrating the intensity and state of polarization of reflected starlight. Note that simplifying the extended disk-integration method to integrating intensities only, thus ignoring polarization, is straightforward.

Basically, for a given planetary phase angle, one can integrate reflected starlight over a planetary disk by calculating the intensity and state of polarization of reflected starlight at various locations across the disk, e.g. assuming a locally plane-parallel planetary atmosphere and/or surface, and using a cubature formula to integrate them (see Horak 1950). For each phase angle, this involves radiative transfer calculations for numerous combinations of the solar zenith angle, viewing zenith angle, and azimuthal angle. In particular when disk-integrated full-flux vectors (fluxes and polarization) are required for a number of phase angles, e.g. to study the planet's phase function, such calculations take huge amounts of computing time. This integration method was used to interpret the polarization of Venus by Hansen \& Hovenier (1974). Monte Carlo simulations for fullflux vectors and various planetary phase angles will also require huge amounts of computing time.
For our disk-integration method, we expand the azimuthaldependent local reflection matrix of the planet (which describes the transformation of a locally incident beam of light into a locally reflected beam for different combinations of solar zenith angles and viewing zenith angles) into a Fourier series. We assume that the planet is spherical, with a plane-parallel atmosphere and/or surface, and that the incident light is unidirectional. The resulting (azimuth-angle-independent) Fourier coefficients are the actual input for the disk-integration algorithm. With these Fourier coefficients, we can directly calculate the coefficients of the expansion of the planetary scattering matrix (which describes the transformation of light that is incident on the planet into light that is reflected back to space) into generalized spherical functions that depend on the planetary phase angle. The expansion coefficients themselves are independent of the planetary phase angle. By substituting the expansion coefficients in the series expansion with the generalized spherical functions, the planetary scattering matrix can be efficiently obtained for any value of the planetary phase angle.

The strong advantage of our disk-integration method is that a single calculation of the local reflection matrix of the planet (for various combinations of solar and viewing zenith angles) suffices to obtain the disk-integrated planetary radiation for any value of the planetary phase angle. We calculate the local reflection of a planet with an adding-doubling algorithm that directly provides the Fourier coefficients of the expansion of the local reflection matrix in the azimuthal angle (de Haan et al. 1987). Obviously, the accuracy of the integration algorithm increases with the number of solar-zenith angles and viewing-zenith angles for which the local reflection matrix is calculated. However, because the computing time also increases with the number of angles, it is up to the user to determine the optimum number of angles. This optimum number will also depend on the reflection properties of the model planet (for example, a planetary model atmosphere that only contains molecules will require fewer angles to reach a given accuracy than one that contains particles with sharp angular features in their single-scattering phase function, see Sect. 4) and the required planetary phase angles.

A disadvantage of our disk-integration method is that in its basic version, it only handles horizontally homogeneous planets, whereas real planets can be inhomogeneous due to e.g. bands of clouds or a patchy surface. However, many types of asymmetries can be simulated by a weighted sum of calculations for different horizontally homogeneous planets, in particular when the planet's rotation is fast compared to the integration time of the observation. For example, a patchy surface can be approximately accounted for by combining calculations for planets with different surface albedos.

The outline of this paper is as follows. In Sect. 2, the numerical disk-integration method is described. In Sect. 3, we test the method by comparing its results with results from independent, e.g. analytical, methods. Section 4 contains sample calculations of the flux and polarization of light reflected by two giant gaseous model planets. For these sample calculations, we used various numbers of solar zenith angles and viewing zenith angles in the calculation of the local reflection matrix, to investigate the influence of the number of angles on the accuracy of the integration for the two different planetary atmospheres and for different phase angles. Section 5 contains the summary and a discussion of the performance of the integration algorithm, both in computational speed and in accuracy. Here, we will also propose a possible further improvement in the efficiency. 


\section{Numerical method}

\subsection{Intensity and flux vectors}

The intensity and state of polarization of a quasi-monochromatic beam of light can be described by an intensity vector $I$ as follows (Chandrasekhar 1950; Hovenier \& van der Mee 1983; Hovenier et al. 2004)

$\boldsymbol{I}=\left[\begin{array}{c}I \\ Q \\ U \\ V\end{array}\right]$

Here, Stokes parameter $I$ is the intensity, $Q$ and $U$ describe the linear polarization, and $V$ describes the circular polarization. All the Stokes parameters of vector $I$ have the dimension $\mathrm{W} \mathrm{m} \mathrm{m}^{-2} \mathrm{sr}^{-1} \mathrm{~Hz}^{-1}$. In this paper, we also use the flux vector $\pi \boldsymbol{F}=\pi[F, Q, U, V]$, of which all Stokes parameters have the dimension $\mathrm{W} \mathrm{m}{ }^{-2} \mathrm{~Hz}^{-1}$.

Parameters $Q$ and $U$ of vectors $\boldsymbol{I}$ and $\pi \boldsymbol{F}$ are defined with respect to a reference plane. In this paper, we use two types of reference planes. First, at each given location on the planet, $Q$ and $U$ are defined with respect to the local meridian plane, i.e. the plane through the local zenith that also contains the direction of propagation of the light. Second, when integrated over the planetary disk, $Q$ and $U$ are defined with respect to the planetary scattering plane, i.e. the plane through the centers of the star and the planet that also contains the observer.

The orientation of the planetary scattering plane with respect to e.g. the optical plane of an Earth-bound polarimeter depends on the orientation of the planetary orbit. Stokes parameters can be transformed from one reference plane to another (e.g. from a planetary scattering plane to a polarimeter's optical plane) using a so-called rotation matrix $\boldsymbol{L}$. This rotation matrix is defined as

$\boldsymbol{L}(\beta)=\left[\begin{array}{cccc}1 & 0 & 0 & 0 \\ 0 & \cos 2 \beta & \sin 2 \beta & 0 \\ 0 & -\sin 2 \beta & \cos 2 \beta & 0 \\ 0 & 0 & 0 & 1\end{array}\right]$.

Angle $\beta$ is the angle between the two reference planes, measured rotating in the anti-clockwise direction from the old to the new reference plane when looking in the direction of propagation $(\beta \geq 0)$.

\subsection{Polarization properties}

From the Stokes parameters, we can derive the polarization properties of the light that is reflected by the planet. Using the parameters of intensity vector $I$ (Eq. (1)) as an example, the total degree of polarization of the reflected starlight is defined as

$P_{\mathrm{t}}=\frac{\sqrt{Q^{2}+U^{2}+V^{2}}}{I}$,

the degree of linear polarization, which we will concentrate on in this paper, as

$P=\frac{\sqrt{Q^{2}+U^{2}}}{I}$,

and the degree of circular polarization as

$P_{\mathrm{c}}=\frac{V}{I}$.

Similar expressions apply for the degree of polarization of flux vector $\pi \boldsymbol{F}$.
The direction of linear polarization with respect to the reference plane, $\chi$, can be found from individual Stokes parameters as follows

$\tan 2 \chi=U / Q$

The convention is to choose the value of $\chi$ in the interval $0 \leq \chi<\pi$, and such that $\cos 2 \chi$ has the same sign as $Q$. Assuming the planet is mirror-symmetric with respect to the planetary scattering plane, the disk-integrated Stokes parameter $U$ will be zero with respect to the scattering plane (Hovenier 1970). In that case and if $Q \neq 0, \chi$ equals either 0 or $\pi / 2$. If $U=0$, we can use an alternative definition of the degree of linear polarization, i.e.

$P_{\mathrm{s}}=-\frac{Q}{I}$

or, for fluxes, $P_{\mathrm{s}}=-Q / F$. With this definition, we add information about the direction of linear polarization. Namely, for $P_{\mathrm{s}}>0$, the light is polarized perpendicular to the planetary scattering plane $(\chi=\pi / 2)$, while for $P_{\mathrm{s}}<0$, it is polarized parallel to this plane $(\chi=0)$. Note that when Stokes parameters are calculated or measured with respect to a reference plane other than the planetary scattering plane, e.g. an instrumental optical plane, $U$ can differ significantly from zero, regardless of any mirrorsymmetry of the planet. We refer to Hovenier et al. (2004) for a more detailed treatment of the subjects in Sects. 2.1 and 2.2.

\subsection{The planetary scattering matrix}

To calculate the observed flux vector of light that has been reflected by a spherical, horizontally homogeneous planet (thus, intensity vectors integrated over the illuminated part of the planetary disk), we describe the reflection process, analogous to scattering by an ensemble of particles, by a matrix multiplication. The vector describing the reflected flux (in $\mathrm{W} \mathrm{m}^{-2} \mathrm{~Hz}^{-1}$ ) as received at a distance $d$ from the planet (where $d$ is much larger than the planet's radius $r$ ), can be written as

$\pi \boldsymbol{F}_{\mathrm{r}}(\Theta)=c \boldsymbol{S}(\Theta) \boldsymbol{F}_{0}$.

Here, $\Theta$ is the total scattering angle $\left(0^{\circ} \leq \Theta \leq 180^{\circ}\right), \pi \boldsymbol{F}_{0}$ the flux vector describing the starlight that is incident on the planet, $S$ the so-called planetary scattering matrix, and $c$ a constant of normalization. The total scattering angle $\Theta$ equals $180^{\circ}-\alpha$, with $\alpha$ the phase angle, i.e. the angle between the star and the observer as seen from the center of the planet. The starlight that is incident on the planetary atmosphere is assumed to be unidirectional, with a flux $\pi F_{0}$ (in $\mathrm{W} \mathrm{m}{ }^{-2} \mathrm{~Hz}^{-1}$ ) measured perpendicularly to the direction of propagation, where $F_{0}$ is the first element of $\boldsymbol{F}_{0}$. Integrated over the stellar disk, the light emitted by solar type stars can be considered to be unpolarized (see e.g. Kemp et al. 1987). In that case, $\boldsymbol{F}_{0}$ is simply described by the vector $F_{0} \mathbf{1}$, with 1 the unit column vector $[1,0,0,0]$. In principle, however, the incident stellar light can have any state of polarization. Also, initially unpolarized stellar light can become polarized before it reaches the planet, such as when it is scattered by circumstellar dust. We therefore describe the disk-integration algorithm for the most general case of arbitrarily polarized incident light.

Using the planetary scattering plane as the reference plane and assuming a planet that is mirror-symmetric with respect to 
this reference plane, the planetary scattering matrix $S$ is given by (see Hovenier 1969, 1970)

$S(\Theta)=\left[\begin{array}{cccc}a_{1}(\Theta) & b_{1}(\Theta) & 0 & 0 \\ b_{1}(\Theta) & a_{2}(\Theta) & 0 & 0 \\ 0 & 0 & a_{3}(\Theta) & b_{2}(\Theta) \\ 0 & 0 & -b_{2}(\Theta) & a_{4}(\Theta)\end{array}\right]$.

Element $a_{1}$ is usually referred to as the planetary phase function. In case the incident light is unpolarized, the only elements of $\boldsymbol{S}$ that are needed to calculate the flux vectors of the light that is reflected by the planet are $a_{1}$ and $b_{1}$ (cf. Eq. (8)). In case the incident light is unpolarized and one is only interested in the reflected flux, only matrix element $a_{1}$ is needed.

The planetary scattering matrix $S$ is normalized so that the average of the planetary phase function over all directions equals the planet's quasi-monochromatic Bond (or spherical) albedo $A_{\mathrm{B}}$, i.e.

$\frac{1}{4 \pi} \int_{4 \pi} a_{1}(\Theta) \mathrm{d} \omega=\frac{1}{2} \int_{0}^{\pi} a_{1}(\Theta) \sin \theta \mathrm{d} \theta \equiv A_{\mathrm{B}}$,

where $\mathrm{d} \omega$ is an element of solid angle. The Bond albedo is defined as the fraction of incident, unpolarized starlight that is reflected by the planet in all directions. Thus, we can also write

$A_{\mathrm{B}} \equiv \frac{d^{2}}{\pi^{2} r^{2} F_{0}} \int_{4 \pi} \pi F_{\mathrm{r}}(\Theta) \mathrm{d} \omega$,

with $r$ the radius of the planet, and $\pi F_{\mathrm{r}}$ the reflected flux, i.e. the first element of flux vector $\pi \boldsymbol{F}_{\mathrm{r}}$. Using Eq. (8), Eq. (11) can be written as

$A_{\mathrm{B}} \equiv \frac{c d^{2}}{\pi^{2} r^{2}} \int_{4 \pi} a_{1}(\Theta) \mathrm{d} \omega$

The normalization constant $c$ now follows from equating Eq. (10) to Eq. (12). We thus find

$\pi \boldsymbol{F}_{\mathrm{r}}(\Theta)=\frac{\pi r^{2}}{4 d^{2}} \boldsymbol{S}(\Theta) \boldsymbol{F}_{0}$

Thus, for a planet with a radius $r$ and with the incident light having a flux vector $\pi \boldsymbol{F}_{0}$, the flux vector of the reflected light as measured at a distance $d$ from the planet can be calculated once the planetary scattering matrix $S$ is known. Note that to calculate the degree and direction of polarization of the reflected light (Eqs. (3)-(7)) only $\boldsymbol{S}$ and $\boldsymbol{F}_{0}$ are required, as $r$ and $d$ drop out of the equations.

To compute the planetary scattering matrix $\boldsymbol{S}$, we express it in terms of the reflection matrix $\boldsymbol{R}$ of a locally plane-parallel planet.

\subsection{The local reflection matrix}

Given a locally plane-parallel and horizontally homogeneous planetary atmosphere containing randomly oriented and optically inactive particles for each of which a mirror particle is present, optionally bounded below by a homogeneous, flat, reflecting surface, the local reflection matrix $\boldsymbol{R}$ can be computed with any appropriate radiative transfer algorithm. We prefer using an efficient algorithm (de Haan et al. 1987) based on the adding-doubling method (Hovenier et al. 2004) that fully includes multiple scattering and polarization.

Directions at the top of the atmosphere are specified by the cosine of the angle $\theta$ between the direction of propagation and the upward vertical, $\mu=|\cos \theta|$, and the azimuthal angle, $\phi$.
The azimuthal angle is measured from an arbitrary vertical plane and clockwise when looking up. The direction of the incident starlight is denoted by $\left(\mu_{0}, \phi_{0}\right)$, with $\mu_{0}=\left|\cos \theta_{0}\right|$, and that of the light that is reflected towards the observer by $(\mu, \phi)$. Since we assume a horizontally homogeneous planet, only the azimuthal difference $\phi-\phi_{0}$ is relevant.

For a given planet model, directions $\mu, \mu_{0}$ and angle $\phi-\phi_{0}$, the intensity vector of the locally reflected starlight, defined with respect to the planetary scattering plane, is given by

$$
\begin{aligned}
\boldsymbol{I}\left(\mu, \mu_{0}, \phi-\phi_{0}\right) & =\mu_{0}, \boldsymbol{L}(\kappa) \boldsymbol{R}\left(\mu, \mu_{0}, \phi-\phi_{0}\right) \boldsymbol{L}\left(\kappa_{0}\right) \boldsymbol{F}_{0} \\
& \equiv \mu_{0} \boldsymbol{R}^{\prime}\left(\mu, \mu_{0}, \phi-\phi_{0}\right) \boldsymbol{F}_{0} .
\end{aligned}
$$

Here, matrix $\boldsymbol{R}$ describes the local reflection of incident starlight, with the local meridian plane (the plane through the local zenith that also contains the direction of propagation of the light) as the reference plane. The matrices $\boldsymbol{L}$ are rotation matrices, which are used to change between reference planes (see Eq. (2). In Eq. (14), $\kappa_{0}$ is the angle of rotation from the planetary scattering plane to the local meridian plane of the incident light, and $\kappa$ is the angle of rotation from the local meridian plane of the reflected light back to the planetary scattering plane. Because the variables $\kappa_{0}$ and $\kappa$ can be expressed in the variables $\theta, \theta_{0}, \phi$ and $\phi_{0}$ (see Sect. 2.6), they have not been written out explicitly in Eq. (15). Note that in the case that the incident starlight is unpolarized, the rotation around $\kappa_{0}$ can be omitted (cf. Eq. (2)). In the case that the incident starlight is unpolarized and one is only interested in the reflected flux, the rotations around $\kappa_{0}$ and $\kappa$ can both be omitted, leaving $\boldsymbol{R}^{\prime}=\boldsymbol{R}$.

The next step towards computing the planetary scattering matrix $S$ is to integrate the intensity vector $\boldsymbol{I}$ of the locally reflected starlight (Eq. (15)) over the illuminated part of the planetary disk.

\subsection{Integration over the planetary disk}

The flux of the reflected light at a distance $d$ from the planet (with $d$ much larger than the planet's radius $r$ ), as described by Eq. (13), follows from integration of the intensity vector of the locally reflected light (Eq. (15)) over the illuminated part of the planetary disk. Thus,

$$
\begin{aligned}
\pi \boldsymbol{F}_{\mathrm{r}}(\Theta) & =\frac{1}{d^{2}} \int_{D} \mu \boldsymbol{I}\left(\mu, \mu_{0}, \phi-\phi_{0}\right) \mathrm{d} O \\
& =\frac{1}{d^{2}} \int_{D} \mu \mu_{0} \boldsymbol{R}^{\prime}\left(\mu, \mu_{0}, \phi-\phi_{0}\right) \boldsymbol{F}_{0} \mathrm{~d} O
\end{aligned}
$$

with $\mu \mathrm{d} O / d^{2}$ the solid angle under which a surface element $\mathrm{d} O$ on the planet is seen by the observer. From Eqs. (13) and (17) we then derive that

$S(\Theta)=\frac{4}{\pi r^{2}} \int_{D} \mu \mu_{0} \boldsymbol{R}^{\prime}\left(\mu, \mu_{0}, \phi-\phi_{0}\right) \mathrm{d} O$.

The integration over the illuminated part of the planetary disk can be performed using the spherical coordinates $\mu$ and $\kappa$ (see Fig. 1). Using $\mathrm{d} O=r^{2} \mathrm{~d} \kappa \mathrm{d} \mu$, Eq. (18) can be written as

$\boldsymbol{S}(\Theta)=\frac{4}{\pi} \int_{0}^{+1} \int_{\kappa^{-}}^{\kappa^{+}} \mu \mu_{0} \boldsymbol{R}^{\prime}\left(\mu, \mu_{0}, \phi-\phi_{0}\right) \mathrm{d} \kappa \mathrm{d} \mu$.

The integration boundaries $\kappa^{ \pm}=\kappa^{ \pm}(\mu, \Theta)$ coincide with the terminator, i.e. the border between day and night that is visible on the planet (see Fig. 1).

Equation (19) can be simplified by recognizing that the hemisphere above the planetary scattering plane (the "northern" 

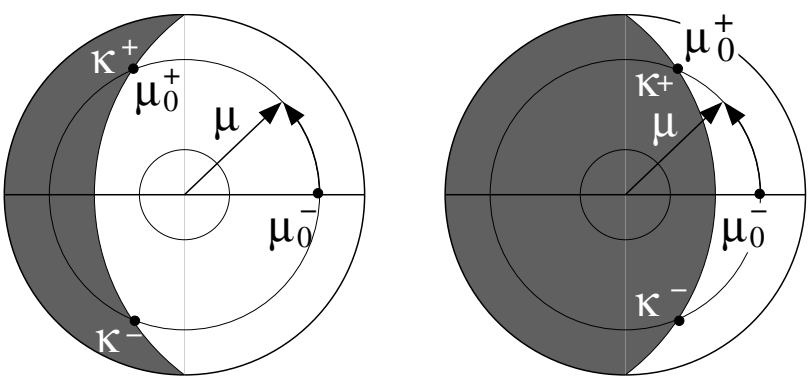

Fig. 1. A sketch of the integration over the illuminated part of the planetary disk as seen by the observer, for a phase angle $\alpha$ of $60^{\circ}$ (on the left) and for $\alpha=120^{\circ}$ (on the right). For the integration, the spherical coordinates $\mu$ and $\kappa$ (see Eq. (19)) or $\mu$ and $\mu_{0}$ (see expression (36)) are used. For $\mu=\cos 50^{\circ}$ (the outer circle drawn within both planetary disks), we indicate the integration boundaries $\kappa^{+}$and $\kappa^{-}$, and $\mu_{0}^{+}$and $\mu_{0}^{-}$(see Eqs. (37) and (38)). For integration along circle $\mu=\cos 50^{\circ}$, $\mu_{0}^{-}=\mu \cos \alpha+\left(1-\mu^{2}\right)^{\frac{1}{2}} \sin \alpha$ and $\mu_{0}^{+}=0$, as the integration stops at the terminator. For integration along the small circle in the disk on the left, $\mu_{0}^{-}=\mu \cos \alpha+\left(1-\mu^{2}\right)^{\frac{1}{2}} \sin \alpha$ and $\mu_{0}^{+}=\mu \cos \alpha-\left(1-\mu^{2}\right)^{\frac{1}{2}} \sin \alpha$. For integration along the small circle in the disk on the right, $\mu_{0}^{-}=0$ and $\mu_{0}^{+}=0$.

hemisphere) is identical to the one below (the "southern" hemisphere). Since each point $X$ on the northern hemisphere corresponds to a point $X^{\prime}$ on the southern hemisphere that is symmetrical with respect to the planetary scattering plane, we have (Hovenier 1970)

$\boldsymbol{R}^{\prime}\left(\mu, \mu_{0}, \phi_{0}-\phi\right)=\Delta \boldsymbol{R}^{\prime}\left(\mu, \mu_{0}, \phi-\phi_{0}\right) \boldsymbol{\Delta}$,

with $\boldsymbol{\Delta}=\operatorname{diag}(1,1,-1,-1)$ (i.e. a matrix with $(1,1,-1,-1)$ along the diagonal and zero's everywhere else). Matrix $S$ of the whole planet can thus easily be obtained from the matrix pertaining to only one, e.g. the northern, hemisphere, using

$S(\Theta)=S_{\mathrm{N}}(\Theta)+\Delta S_{\mathrm{N}}(\Theta) \Delta$,

where $S_{\mathrm{N}}$ is the matrix of the northern hemisphere, given by (see Eq. (19))

$S_{\mathrm{N}}(\Theta)=\frac{4}{\pi} \int_{0}^{+1} \int_{0}^{\kappa^{+}} \mu \mu_{0} \boldsymbol{R}^{\prime}\left(\mu, \mu_{0}, \phi-\phi_{0}\right) \mathrm{d} \kappa \mathrm{d} \mu$.

In principle, we can thus (i) calculate $\boldsymbol{R}$ using a plane-parallel model planetary atmosphere, for various combinations of $\mu, \mu_{0}$, and angle $\phi-\phi_{0}$, (ii) calculate $\boldsymbol{R}^{\prime}$ for different locations on the planet, (iii) evaluate Eq. (22) for a given value of $\Theta$, and finally (iv) use Eq. (21) to obtain the planetary scattering matrix $S(\Theta)$. In practice, a numerically more efficient algorithm can be used as described in detail in the next sections. In Sect. 2.11, this algorithm is summarized in the form of a numerical recipe comprising 5 practical steps.

\subsection{Expansion in generalized spherical functions}

Instead of using Eqs. (20)-(22), a more efficient approach numerically is to calculate the coefficients for the expansion of the elements of matrix $\boldsymbol{S}$ into so-called generalized spherical functions (see Gel'fand et al. 1963; Hovenier \& van der Mee 1983; Hovenier et al. 2004). As seen later, once these expansion coefficients are available, the elements of the planetary scattering matrix can easily and efficiently be calculated for any value of the total scattering angle $\Theta$. This expansion method was originally proposed by van de Hulst (1980) for the scalar case (thus, without polarization).
The calculation of the expansion coefficients appears to be started best with a complex notation for the intensity and flux vectors and scattering matrices. In the complex notation (indicated by a hat), an intensity vector is given by

$\hat{\boldsymbol{I}} \equiv\left[\begin{array}{c}\hat{I}_{+2} \\ \hat{I}_{+0} \\ \hat{I}_{-0} \\ \hat{I}_{-2}\end{array}\right]=\frac{1}{2}\left[\begin{array}{c}Q+\mathrm{i} U \\ I+V \\ I-V \\ Q-\mathrm{i} U\end{array}\right]=\frac{1}{2}\left[\begin{array}{cccc}0 & 1 & \mathrm{i} & 0 \\ 1 & 0 & 0 & 1 \\ 1 & 0 & 0 & -1 \\ 0 & 1 & -\mathrm{i} & 0\end{array}\right]\left[\begin{array}{c}I \\ Q \\ U \\ V\end{array}\right] \equiv \boldsymbol{T I}$

Note that the indices run as $+2,+0,-0,-2$. Using $\hat{\boldsymbol{F}}_{\mathrm{r}}=\boldsymbol{T} \boldsymbol{F}_{\mathrm{r}}$ (the flux version of Eq. (23) and Eq. (13)), the relation between $\boldsymbol{S}$ and $\hat{\boldsymbol{S}}$ is found to be

$\hat{\boldsymbol{S}}(\Theta)=\boldsymbol{T S}(\Theta) \boldsymbol{T}^{-1}$ or $\boldsymbol{S}(\Theta)=\boldsymbol{T}^{-1} \hat{\boldsymbol{S}}(\Theta) T$.

The inverse matrix $\boldsymbol{T}^{-1}$ is given by

$\boldsymbol{T}^{-1}=\left[\begin{array}{cccc}0 & 1 & 1 & 0 \\ 1 & 0 & 0 & 1 \\ -\mathrm{i} & 0 & 0 & \mathrm{i} \\ 0 & 1 & -1 & 0\end{array}\right]$

The next step towards the expansion coefficients of $\boldsymbol{S}$ is the expansion of the elements of the complex planetary scattering matrix $\hat{\boldsymbol{S}}$ in generalized spherical functions. This expansion reads

$\hat{S}_{k, n}(\Theta)=\sum_{l=\max (|k|,|n|)}^{\infty} \hat{S}_{k, n}^{l} P_{k, n}^{l}(\cos \Theta)$

with the indices $k$ and $n$ equal to $+2,+0,-0$, or -2 . Here, the $\hat{S}_{k, n}^{l}$ are elements of the complex expansion coefficients matrix $\hat{\boldsymbol{S}}^{l}$, and the $P_{k, n}^{l}$ are generalized spherical functions (see Gel'fand et al. 1963; Hovenier \& van der Mee 1983; Hovenier et al. 2004), for which there is no difference between the indices +0 and -0 . They satisfy the relations

$P_{k, n}^{l}(x)=P_{-k,-n}^{l}(x)=P_{n, k}^{l}(x)=(-1)^{l+k-n} P_{-k, n}^{l}(-x)$.

Due to the reciprocity and mirror symmetry of the reflection process, the complex expansion coefficients themselves obey the following symmetry relations

$\hat{S}_{k, n}^{l}=\hat{S}_{n, k}^{l}=\hat{S}_{-k,-n}^{l}$.

In order to calculate the complex expansion coefficients matrix $\hat{\boldsymbol{S}}^{l}$ (which will then be substituted in Eq. (24)), we next apply the following orthogonality relation for generalized spherical functions (Eq. (B.9) in Hovenier et al. 2004) to both sides of Eq. (26)

$(-1)^{k+n} \int_{-1}^{+1} P_{k, n}^{l}(x) P_{k, n}^{j}(x) \mathrm{d}(x)=\frac{2}{2 l+1} \delta_{l j}$,

with $\delta_{l j}$ the Kronecker delta function. We thus transform Eq. (26) into

$\hat{S}_{k, n}^{l}=\left(l+\frac{1}{2}\right) \int_{-1}^{+1} P_{k, n}^{l}(\cos \Theta) \hat{S}_{k, n}(\Theta) \mathrm{d}(\cos \Theta)$.

By using $\hat{\boldsymbol{R}}^{\prime}=\boldsymbol{T} \boldsymbol{R}^{\prime} \boldsymbol{T}^{\mathbf{1}}$ (cf. Eq. (24)) we next obtain the complex expression of Eq. (19), which describes the relation between the planetary scattering matrix $S$ and the local reflection matrix $\boldsymbol{R}^{\prime}$. Inserting this complex expression into Eq. (30) and writing $u=\cos \Theta$ then give

$\hat{S}_{k, n}^{l}=\frac{4 l+2}{\pi} \int_{-1}^{+1} \int_{0}^{+1} \int_{\kappa^{-}}^{\kappa^{+}} \hat{B}_{k, n}^{l}\left(u, \mu, \mu_{0}, \phi-\phi_{0}\right) \mu \mu_{0} \mathrm{~d} \kappa \mathrm{d} \mu \mathrm{d} u$ 
where $\hat{B}_{k, n}^{l}\left(u, \mu, \mu_{0}, \phi-\phi_{0}\right)$ stands for $P_{k, n}^{l}(u) \hat{R}_{k, n}^{\prime}\left(\mu, \mu_{0}, \phi-\phi_{0}\right)$. Next, we want to change to integrals over $\mu, \mu_{0}$, and $\phi-\phi_{0}$, since we assume that the local reflection matrix $\boldsymbol{R}$ has been calculated for various combinations of these variables. Therefore, we first write the "northern hemisphere" part of the triple integral in Eq. (31) as (cf. Eq. (22))

$$
\int_{-1}^{+1} \int_{0}^{+1} \int_{0}^{\kappa^{+}} \hat{B}_{k, n}^{l}\left(u, \mu, \mu_{0}, \phi-\phi_{0}\right) \mu \mu_{0} \mathrm{~d} \kappa \mathrm{d} \mu \mathrm{d} u .
$$

Now, the integration over variable $\kappa$ is replaced by an integration over $\mu_{0}$. Using spherical trigonometry, we can derive (see Fig. 1) that

$\sin \kappa=\left(1-\mu_{0}^{2}\right)^{\frac{1}{2}} \frac{\sin \left(\phi-\phi_{0}\right)}{\sin \Theta}$

and that

$\mu_{0}=-\mu \cos \Theta+\left(1-\mu^{2}\right)^{\frac{1}{2}} \sin \Theta \cos \kappa$.

Thus,

$\frac{\partial \mu_{0}}{\partial \kappa}=-\left(1-\mu^{2}\right)^{\frac{1}{2}}\left(1-\mu_{0}^{2}\right)^{\frac{1}{2}} \sin \left(\phi-\phi_{0}\right)$.

When also changing from variable $u=\cos \Theta$ to $\cos \alpha$, with $\alpha$ the phase angle of the planet, Eq. (32) can be written as

$\int_{-1}^{+1} \int_{0}^{+1} \int_{\mu_{0}^{-}}^{\mu_{0}^{+}} \hat{B}_{k, n}^{l}\left(u, \mu, \mu_{0}, \phi-\phi_{0}\right) \frac{\partial \kappa}{\partial \mu_{0}} \mu \mu_{0} \mathrm{~d} \mu_{0} \mathrm{~d} \mu \mathrm{d} \cos \alpha$.

The integration boundaries $\mu_{0}^{-}$and $\mu_{0}^{+}$depend on $\alpha$ and $\mu$. The following cases can be distinguished (see Fig. 1)

$\mu_{0}^{-}=\left\{\begin{array}{lll}0 & \text { if } & \cos \alpha \leq-\left(1-\mu^{2}\right)^{\frac{1}{2}} \\ \mu \cos \alpha+\left(1-\mu^{2}\right)^{\frac{1}{2}} \sin \alpha & \text { if } & \cos \alpha>-\left(1-\mu^{2}\right)^{\frac{1}{2}}\end{array}\right.$
$\mu_{0}^{+}=\left\{\begin{array}{lll}0 & \text { if } & \cos \alpha \leq\left(1-\mu^{2}\right)^{\frac{1}{2}} \\ \mu \cos \alpha-\left(1-\mu^{2}\right)^{\frac{1}{2}} \sin \alpha & \text { if } & \cos \alpha>\left(1-\mu^{2}\right)^{\frac{1}{2}}\end{array}\right.$

(note that when $\mu_{0}^{-}=0, \mu_{0}^{+}$will also be 0 ). We will now replace the integration over $\cos \alpha$ in Eq. (36) by an integration over $\left(\phi-\phi_{0}\right)$, using

$\cos \alpha=\mu \mu_{0}-\left(1-\mu^{2}\right)^{\frac{1}{2}}\left(1-\mu_{0}^{2}\right)^{\frac{1}{2}} \cos \left(\phi-\phi_{0}\right)$.

Thus (see also Eq. (35)),

$\frac{\partial \cos \alpha}{\partial\left(\phi-\phi_{0}\right)}=\left(1-\mu^{2}\right)^{\frac{1}{2}}\left(1-\mu_{0}^{2}\right)^{\frac{1}{2}} \sin \left(\phi-\phi_{0}\right) \equiv-\frac{\partial \mu_{0}}{\partial \kappa}$

and, having started from Eq. (31), we finally arrive at the following expression for the elements of the complex expansion coefficients matrix $\hat{\boldsymbol{S}}^{l}$

$$
\begin{aligned}
\hat{S}_{k, n}^{l} & =\frac{4 l+2}{\pi} \\
& \times \int_{0}^{2 \pi} \int_{0}^{+1} \int_{0}^{+1} \hat{B}_{k, n}^{l}\left(u, \mu, \mu_{0}, \phi-\phi_{0}\right) \mu \mu_{0} \mathrm{~d} \mu_{0} \mathrm{~d} \mu \mathrm{d}\left(\phi-\phi_{0}\right),
\end{aligned}
$$

with $\hat{B}_{k, n}^{l}\left(u, \mu, \mu_{0}, \phi-\phi_{0}\right)=P_{k, n}^{l}(u) \hat{R}_{k, n}^{\prime}\left(\mu, \mu_{0}, \phi-\phi_{0}\right)$. In Eq. (41), we have extended the integration over $\left(\phi-\phi_{0}\right)$ from $[0, \pi]$ to $[0,2 \pi]$ to include the southern hemisphere of the planet. Expansion coefficients $\hat{S}_{k, n}^{l}$ thus pertain to the whole illuminated part of the planetary disk. In the next section, we demonstrate how the integral in Eq. (41) can be simplified by expanding the complex local reflection matrix $\hat{\boldsymbol{R}}^{\prime}$ into a Fourier series.

\subsection{Expansion in Fourier coefficients}

The expansion of the complex local reflection matrix $\hat{\boldsymbol{R}}^{\prime}$ into a Fourier series is started with writing $\hat{\boldsymbol{R}}^{\prime}$ as (see Eqs. (24) and (15))

$$
\begin{aligned}
\hat{\boldsymbol{R}}^{\prime}\left(\mu, \mu_{0}, \phi-\phi_{0}\right) & =\boldsymbol{T} \boldsymbol{R}^{\prime}\left(\mu, \mu_{0}, \phi-\phi_{0}\right) \boldsymbol{T}^{-1} \\
& =\boldsymbol{T} \boldsymbol{L}(\kappa) \boldsymbol{R}\left(\mu, \mu_{0}, \phi-\phi_{0}\right) \boldsymbol{L}\left(\kappa_{0}\right) \boldsymbol{T}^{-1} \\
& \equiv \hat{\boldsymbol{L}}(\kappa) \hat{\boldsymbol{R}}\left(\mu, \mu_{0}, \phi-\phi_{0}\right) \hat{\boldsymbol{L}}\left(\kappa_{0}\right),
\end{aligned}
$$

with $\hat{\boldsymbol{L}}=\boldsymbol{T} \boldsymbol{L} \boldsymbol{T}^{-1}$ and $\hat{\boldsymbol{R}}=\boldsymbol{T} \boldsymbol{R} \boldsymbol{T}^{-1}$. In the complex notation, a rotation matrix $\hat{\boldsymbol{L}}$ has a convenient diagonal form, with elements $\hat{L}_{k, n}(\beta)=\exp (-\mathrm{i} k \beta) \delta_{k, n}$ (with $k$ equal to $+2,+0,-0$, or -2). Using Eq. (44), each matrix element $\hat{R}_{k, n}^{\prime}$ in Eq. (41) can thus be replaced by

$\hat{R}_{k, n}^{\prime}\left(\mu, \mu_{0}, \phi-\phi_{0}\right)=\mathrm{e}^{-\mathrm{i} k \kappa} \hat{R}_{k, n}\left(\mu, \mu_{0}, \phi-\phi_{0}\right) \mathrm{e}^{-\mathrm{i} n \kappa_{0}}$.

The complex matrix $\hat{\boldsymbol{R}}$ can be expanded into a Fourier series as follows

$\hat{\boldsymbol{R}}\left(\mu, \mu_{0}, \phi-\phi_{0}\right)=\sum_{s=-\infty}^{+\infty} \hat{\boldsymbol{R}}^{s}\left(\mu, \mu_{0}\right) \mathrm{e}^{-\mathrm{i} s\left(\phi-\phi_{0}\right)}$.

Here, $\hat{\boldsymbol{R}}^{s}$ denotes Fourier coefficient $s$. Next, we insert Eqs. (45) and (46) into Eq. (41), and invoke the addition theorem for the generalized spherical functions (Eq. (B.48) in Hovenier et al. 2004). After integration over the azimuthal angle $\left(\phi-\phi_{0}\right)$, we then arrive at

$$
\begin{aligned}
\hat{S}_{k, n}^{l}= & 4(2 l+1) \\
& \times \sum_{s=-l}^{l}(-1)^{s} \int_{0}^{+1} \int_{0}^{+1} P_{n, s}^{l}(-\mu) \hat{R}_{k, n}^{s}\left(\mu, \mu_{0}\right) P_{s, k}^{l}\left(\mu_{0}\right) \mu \mu_{0} \mathrm{~d} \mu_{0} \mathrm{~d} \mu .
\end{aligned}
$$

Defining matrices $\boldsymbol{P}_{s}^{l}(u)=\operatorname{diag}\left(P_{s, 2}^{l}(u), P_{s, 0}^{l}(u), P_{s, 0}^{l}(u), P_{s,-2}^{l}(u)\right)$ (here, we used $P_{s,-0}^{l}=P_{s, 0}^{l}$, according to Eq. (27)), the complex expansion coefficients matrices can hence be written as

$\hat{\boldsymbol{S}}^{l}=4(2 l+1)$

$$
\times \sum_{s=-l}^{l}(-1)^{s} \int_{0}^{+1} \int_{0}^{+1} \boldsymbol{P}_{s}^{l}(-\mu) \hat{\boldsymbol{R}}^{s}\left(\mu, \mu_{0}\right) \boldsymbol{P}_{s}^{l}\left(\mu_{0}\right) \mu \mu_{0} \mathrm{~d} \mu_{0} \mathrm{~d} \mu .
$$

For computational purposes it is desirable to rewrite the complex expansion coefficients matrices of the planetary scattering matrix into real ones. This will be done in the next section.

\subsection{Real expansion coefficients matrices}

To derive the relations between the complex and the real expansion coefficients, we start with the latter. The matrix elements of the real planetary scattering matrix $S$ (see Eq. (9)) can be expanded in the following series

$$
\begin{gathered}
a_{1}(\Theta)=\sum_{l=0}^{\infty} \alpha_{1}^{l} P_{0,0}^{l}(\cos \Theta) . \\
a_{2}(\Theta)+a_{3}(\Theta)=\sum_{l=2}^{\infty}\left(\alpha_{2}^{l}+\alpha_{3}^{l}\right) P_{2,2}^{l}(\cos \Theta) . \\
a_{2}(\Theta)-a_{3}(\Theta)=\sum_{l=2}^{\infty}\left(\alpha_{2}^{l}-\alpha_{3}^{l}\right) P_{2,-2}^{l}(\cos \Theta) .
\end{gathered}
$$




$$
\begin{aligned}
& a_{4}(\Theta)=\sum_{l=0}^{\infty} \alpha_{4}^{l} P_{0,0}^{l}(\cos \Theta) . \\
& b_{1}(\Theta)=\sum_{l=2}^{\infty} \beta_{1}^{l} P_{0,2}^{l}(\cos \Theta) . \\
& b_{2}(\Theta)=\sum_{l=2}^{\infty} \beta_{2}^{l} P_{0,2}^{l}(\cos \Theta) .
\end{aligned}
$$

The real expansion coefficients are elements of the real expansion coefficients matrix $S^{l}$, as follows

$\boldsymbol{S}^{l}=\left[\begin{array}{cccc}\alpha_{1}^{l} & \beta_{1}^{l} & 0 & 0 \\ \beta_{1}^{l} & \alpha_{2}^{l} & 0 & 0 \\ 0 & 0 & \alpha_{3}^{l} & \beta_{2}^{l} \\ 0 & 0 & -\beta_{2}^{l} & \alpha_{4}^{l}\end{array}\right]$.

By explicit multiplication of the matrices in Eq. (24) and by using the relations from Eqs. (27) and (28), the relations between the complex and the real expansion coefficients can be derived. We thus find

$\alpha_{1}^{l}=\hat{S}_{0,0}^{l}+\hat{S}_{0,-0}^{l}$.

$\alpha_{2}^{l}=\hat{S}_{2,2}^{l}+\hat{S}_{2,-2}^{l}$.

$\alpha_{3}^{l}=\hat{S}_{2,2}^{l}-\hat{S}_{2,-2}^{l}$.

$\alpha_{4}^{l}=\hat{S}_{0,0}^{l}-\hat{S}_{0,-0}^{l}$.

$\beta_{1}^{l}=\hat{S}_{0,2}^{l}+\hat{S}_{0,-2}^{l}$.

$\beta_{2}^{l}=-i\left(\hat{S}_{0,2}^{l}-\hat{S}_{0,-2}^{l}\right)$.

The relations between the real expansion coefficient matrices $\boldsymbol{S}^{l}$ and the complex ones $\hat{\boldsymbol{S}}^{l}$ (see Eq. (49)) as expressed with the respective expansion coefficients in Eqs. (56)-(61), are described by the following matrix product

$\boldsymbol{S}^{l}=\frac{1}{2}(\mathbf{1}+\mathrm{i} \boldsymbol{\Delta}) \boldsymbol{T}^{-1} \hat{\boldsymbol{S}}^{l} \boldsymbol{T}(\mathbf{1}-\mathrm{i} \boldsymbol{\Delta})$

(recall that $\boldsymbol{\Delta}=\operatorname{diag}(1,1,-1,-1)$ ). Substitution of Eq. (49) into Eq. (62) will lead to the relation between the real and the complex expansion coefficients matrices. Before we do that, however, we replace the complex Fourier coefficients matrices $\hat{\boldsymbol{R}}^{s}$ that appear in Eq. (49) by real matrices. The relation between the complex and the real Fourier coefficients matrices is derived in the next section.

\subsection{Real Fourier coefficient matrices}

Using $\boldsymbol{R}=\boldsymbol{T}^{-1} \hat{\boldsymbol{R}} \boldsymbol{T}$ (Eq. (24)), Eq. (46), and the following real Fourier series expansion (Hovenier 1971)

$$
\begin{aligned}
& \boldsymbol{R}\left(\mu, \mu_{0}, \phi-\phi_{0}\right)=\sum_{s=0}^{+\infty}\left(2-\delta_{s, 0}\right) \\
& \quad \times\left[\boldsymbol{R}_{\mathrm{c}}^{s}\left(\mu, \mu_{0}\right) \cos s\left(\phi-\phi_{0}\right)+\boldsymbol{R}_{\mathrm{s}}^{s}\left(\mu, \mu_{0}\right) \sin s\left(\phi-\phi_{0}\right)\right],
\end{aligned}
$$

where the $4 \times 4$ matrix $\boldsymbol{R}_{\mathrm{c}}^{s}\left(\boldsymbol{R}_{\mathrm{s}}^{s}\right)$ has $2 \times 2$ zero submatrices in the upper (lower) right and lower (upper) left corners, and where $\boldsymbol{R}_{\mathrm{s}}^{0}$ is chosen equal to zero, we find that

$$
\begin{aligned}
\boldsymbol{R}_{\mathrm{c}}^{s} & =\frac{1}{2}\left[\boldsymbol{T}^{-1} \hat{\boldsymbol{R}}^{-s} \boldsymbol{T}+\boldsymbol{T}^{-1} \hat{\boldsymbol{R}}^{s} \boldsymbol{T}\right] . \\
\boldsymbol{R}_{\mathrm{s}}^{s} & =\frac{i}{2}\left[\boldsymbol{T}^{-1} \hat{\boldsymbol{R}}^{-s} \boldsymbol{T}-\boldsymbol{T}^{-1} \hat{\boldsymbol{R}}^{s} \boldsymbol{T}\right] .
\end{aligned}
$$

Here we have omitted the explicit $\left(\mu, \mu_{0}\right)$ dependence of the Fourier coefficients matrices. Writing (see de Haan et al. 1987)

$\boldsymbol{R}^{s}=\boldsymbol{R}_{\mathrm{c}}^{s}-\Delta \boldsymbol{R}_{\mathrm{s}}^{s}$

we get

$$
\boldsymbol{R}^{s}=\frac{1}{2}(\mathbf{1}-\mathrm{i} \Delta) \boldsymbol{T}^{-1} \hat{\boldsymbol{R}}^{-s} \boldsymbol{T}+\frac{1}{2}(\mathbf{1}+\mathrm{i} \boldsymbol{\Delta}) \boldsymbol{T}^{-1} \hat{\boldsymbol{R}}^{s} \boldsymbol{T}
$$

This equation can be simplified by combining the following relation (see the symmetry relations given by Hovenier 1969)

$\boldsymbol{R}\left(\mu, \mu_{0}, \phi-\phi_{0}\right)=\Delta \boldsymbol{R}\left(\mu, \mu_{0}, \phi_{0}-\phi\right) \boldsymbol{\Delta}$,

with (see Eq. (46))

$\boldsymbol{R}\left(\mu, \mu_{0}, \phi-\phi_{0}\right)=\sum_{s=-\infty}^{+\infty} \boldsymbol{T}^{-1} \hat{\boldsymbol{R}}^{s} \boldsymbol{T} \mathrm{e}^{-\mathrm{i} s\left(\phi-\phi_{0}\right)}$.

We thus find

$\boldsymbol{T}^{-1} \hat{\boldsymbol{R}}^{-s} \boldsymbol{T}=\Delta \boldsymbol{T}^{-1} \hat{\boldsymbol{R}}^{s} \boldsymbol{T} \Delta$.

Substituting Eq. (70) into Eq. (67) and using $\frac{1}{2}(\mathbf{1}-\mathrm{i} \boldsymbol{\Delta}) \times(\mathbf{1}+\mathrm{i} \boldsymbol{\Delta})=$ 1 yields the following expressions for the Fourier coefficients matrices of the complex reflection matrices $\hat{\boldsymbol{R}}$

$$
\begin{aligned}
\hat{\boldsymbol{R}}^{s} & =\frac{1}{2} \boldsymbol{T}(\mathbf{1}-\mathrm{i} \Delta) \boldsymbol{R}^{s}(\mathbf{1}+\mathrm{i} \Delta) \boldsymbol{T}^{-1} . \\
\hat{\boldsymbol{R}}^{-s} & =\frac{1}{2} \boldsymbol{T}(\mathbf{1}-\mathrm{i} \Delta) \Delta \boldsymbol{R}^{s} \Delta(\mathbf{1}+\mathrm{i} \Delta) T^{-1} .
\end{aligned}
$$

The substitution of these expressions into Eq. (49) will be described in the next section.

\subsection{The final expression}

The substitution of Eqs. (49), (71), and (72) into Eq. (62) gives an elaborate expression that can be made more readable by defining yet another type of matrix, namely $\boldsymbol{Q}_{s}^{l}(u)=\frac{1}{2}(\mathbf{1}+\mathrm{i} \boldsymbol{\Delta}) \boldsymbol{T}^{-1} \boldsymbol{P}_{s}^{l}(u) \boldsymbol{T}(\mathbf{1}-\mathrm{i} \boldsymbol{\Delta})$, or

$\boldsymbol{Q}_{s}^{l}(u)=$

$$
\frac{1}{2}\left[\begin{array}{cccc}
2 P_{s, 0}^{l}(u) & 0 & 0 & 0 \\
0 & P_{s,-2}^{l}(u)+P_{s, 2}^{l}(u) & P_{s,-2}^{l}(u)-P_{s, 2}^{l}(u) & 0 \\
0 & P_{s,-2}^{l}(u)-P_{s, 2}^{l}(u) & P_{s,-2}^{l}(u)+P_{s, 2}^{l}(u) & 0 \\
0 & 0 & 0 & 2 P_{s, 0}^{l}(u)
\end{array}\right] .
$$

From Eq. (27), it follows that

$\boldsymbol{Q}_{s}^{l}(u)=\Delta \boldsymbol{Q}_{-s}^{l}(u) \Delta$.

Substituting Eqs. (49), (71), (72), (73), and (74) into Eq. (62) gives the following final expression for the real planetary expansion coefficients matrices

$\boldsymbol{S}^{l}=(2 l+1)\left[\boldsymbol{R}_{l}^{0}+\sum_{s=1}^{l}(-1)^{s}\left(\boldsymbol{R}_{l}^{s}+\Delta \boldsymbol{R}_{l}^{s} \boldsymbol{\Delta}\right)\right]$,

where the matrices $\boldsymbol{R}_{l}^{s}$ are defined by

$\boldsymbol{R}_{l}^{s}=4 \int_{0}^{+1} \int_{0}^{+1} \boldsymbol{Q}_{s}^{l}(-\mu) \boldsymbol{R}^{s}\left(\mu, \mu_{0}\right) \boldsymbol{Q}_{s}^{l}\left(\mu_{0}\right) \mu \mu_{0} \mathrm{~d} \mu_{0} \mathrm{~d} \mu$.

Here, the matrices $\boldsymbol{R}^{s}$ are the Fourier coefficients matrices of the local reflection matrix $\boldsymbol{R}$ (Eq. (67)). With the $\boldsymbol{R}_{l}^{s}$ and $\boldsymbol{S}^{l}$, the 
elements of the planetary scattering matrix $S$ can be obtained from Eqs. (49)-(54) and Eq. (55).

In case the incident starlight is unpolarized, only the elements $a_{1}$ and $b_{1}$ of $\boldsymbol{S}$ (Eq. (9)) are needed to calculate the flux vector and the degree of polarization of the light that is reflected by the planet. This means that in that case only the elements $\alpha_{1}^{l}$ and $\beta_{1}^{l}$ of the expansion coefficients matrices $\boldsymbol{S}^{l}$ are needed, which implies that only the first column of the matrices $\boldsymbol{R}_{l}^{s}$, and thus only the first column of $\boldsymbol{R}^{s}$ (see Eqs. (76) and (73)), has to be calculated. In case the incident starlight is unpolarized and one is only interested in the reflected flux, only the $(1,1)$-elements of these matrices are needed. Warning: even if only the (1,1)-element of the local reflection matrix $\boldsymbol{R}$ of a planetary atmosphere is needed, polarization should be taken into account for calculating this element when there is some fraction of the light that is multiple scattered. Otherwise, errors up to several percent can be introduced in the calculated disk-integrated reflected flux and related quantities like the geometric albedo (Sromovsky 2005; Stam \& Hovenier 2005).

\subsection{Numerical recipe}

For a given model of a planetary atmosphere, the elements of the planetary scattering matrix $S(\Theta)$ can thus be obtained using the following recipe:

1. Calculate the local reflection matrix $\boldsymbol{R}\left(\mu, \mu_{0}, \phi-\phi_{0}\right)$ using for example an adding-doubling algorithm.

2. Use Eqs. (64) and (66) to find the Fourier coefficients matrices $\boldsymbol{R}^{s}\left(\mu, \mu_{0}\right)$.

3. Compute the matrices $\boldsymbol{R}_{l}^{s}$ from Eqs. (73) and (76).

4. Compute the planetary expansion coefficients matrices $\boldsymbol{S}^{l}$ using Eq. (75), which yields the expansion coefficients $\alpha_{1}^{l}, \alpha_{2}^{l}$, $\alpha_{3}^{l}, \alpha_{4}^{l}, \beta_{1}^{l}$, and $\beta_{2}^{l}$ (see Eq. (55)).

5. Using Eqs. (49)-(54), all elements of the planetary scattering matrix $S$ (see Eq. (9)) can then be calculated for any value of the total scattering angle $\Theta$ or phase angle $\alpha$.

It is important to note that the calculation of the local reflection matrix $\boldsymbol{R}$ (step 1) can be done with an arbitrary radiative transfer algorithm. We prefer to use the adding-doubling algorithm as described by (de Haan et al. 1987), which directly yields the Fourier coefficients matrices $\boldsymbol{R}^{s}$ (step 2).

We perform the integrations in Eq. (76) (step 3) using GaussLegendre quadrature (see e.g. Press et al. 1986). For this integration, the values of $\mu$ and $\mu_{0}$ at which the $\boldsymbol{R}^{s}$ are calculated are chosen at Gauss-Legendre quadrature abscissae. Thus, for an integration with $M$ abscissae, the Fourier coefficients are calculated at an $M \times M$ grid of $\left(\mu, \mu_{0}\right)$-pairs. The number $M$ required to reach a given integration accuracy will strongly depend on the composition and structure of the planetary model atmosphere, because the smoother the local reflection matrix $\boldsymbol{R}$, the fewer abscissae will be needed. Because the smoothness of the local reflection matrix generally varies with $\mu, \mu_{0}$ and $\phi-\phi_{0}$, the appropriate $M$ will also depend on the total scattering angle $\Theta$ (or phase angle $\alpha$ ). The accuracy of the disk-integration algorithm and its dependence on $M$ will be explored in Sect. 3 .

When calculating the elements of the planetary scattering matrix (step 5), the value of $l$ where the summations in Eqs. (49)-(54) should be broken off has to be chosen carefully. Typically, the absolute value of the expansion coefficients starts with a sharp increase with $l$, but then decreases steadily with $l$, until a loss of numerical accuracy leads to a rapid increase in the absolute value of the coefficients. We thus break off a summation just before the absolute values of the expansion coefficients start to increase again.

\subsection{Related quantities}

From the planetary scattering matrix $S$ and its expansion coefficients, various useful, related quantities can be calculated, such as the Bond albedo, the geometric albedo, and the phase integral of the planet.

The Bond albedo is given by (see Eqs. (10)-(13) and Eq. (49))

$$
\begin{aligned}
A_{\mathrm{B}} & =\frac{d^{2}}{\pi r^{2} \pi F_{0}} \int_{4 \pi} F_{\mathrm{r}}(\Theta) \mathrm{d} \omega \\
& =\frac{1}{4 \pi} \int_{0}^{2 \pi} \int_{0}^{\pi} a_{1}(\Theta) \sin \Theta \mathrm{d} \Theta \mathrm{d} \phi \\
& =\frac{1}{2} \sum_{l=0}^{\infty} \int_{0}^{\pi} \alpha_{1}^{l} P_{0,0}^{l}(\cos \Theta) \sin \Theta \mathrm{d} \Theta \\
& =\alpha_{1}^{0} .
\end{aligned}
$$

The Bond albedo thus equals the $(1,1)$-element of the expansion coefficients matrix $S^{0}$ (see Eq. (55)) (see the warning at the end of Sect. 2.10).

The geometric albedo $p$ of a planet is the ratio of the observed flux of the planet (see Eq. (13)) when the phase angle $\alpha$ equals $0^{\circ}$ (or $\Theta=180^{\circ}$ ) to the observed flux of a Lambertian surface (i.e. a surface that reflects all incoming radiation isotropically and completely depolarized) that receives the same incoming flux and that subtends the same solid angle (i.e. $\pi r^{2} / d^{2}$, with $r$ the planetary radius and $d$ the distance between the planet and the observer) on the sky. Thus,

$p=\frac{F_{\mathrm{r}}\left(180^{\circ}\right) d^{2}}{F_{0} \pi r^{2}}=\frac{1}{4} a_{1}\left(180^{\circ}\right)$,

with $a_{1}$ the (1,1)-element of the planetary scattering matrix $S$ (see Eq. (9)), which is also called the planetary phase function.

The planetary phase function can be renormalized such that it equals one when the phase angle is zero (at $\Theta=180^{\circ}$ ). The integral of this normalized, so-called classical phase function over all scattering directions times two defines the phase integral, which is usually denoted by $q$. Thus,

$q=2 \int_{0}^{\pi} \frac{a_{1}(\Theta) \sin \Theta}{a_{1}\left(180^{\circ}\right)} \mathrm{d} \Theta$.

The phase integral equals the ratio of the Bond albedo to the geometric albedo, thus $q=A_{\mathrm{B}} / p$.

Note that in this paper we have assumed quasimonochromatic radiation. When comparing results of numerical simulations with observations, such wavelength dependence should, of course, be accounted for.

\section{Numerical tests}

To test the accuracy of our disk integration algorithm, we compare planetary scattering matrices as obtained with our numerical integration method with those obtained with other methods. Because in most applications the starlight that is incident on the planetary atmosphere will be assumed to be unpolarized, we will only describe the tests for the elements $a_{1}$ and $b_{1}$ of the planetary scattering matrix $S$ (see Eq. (9)). 


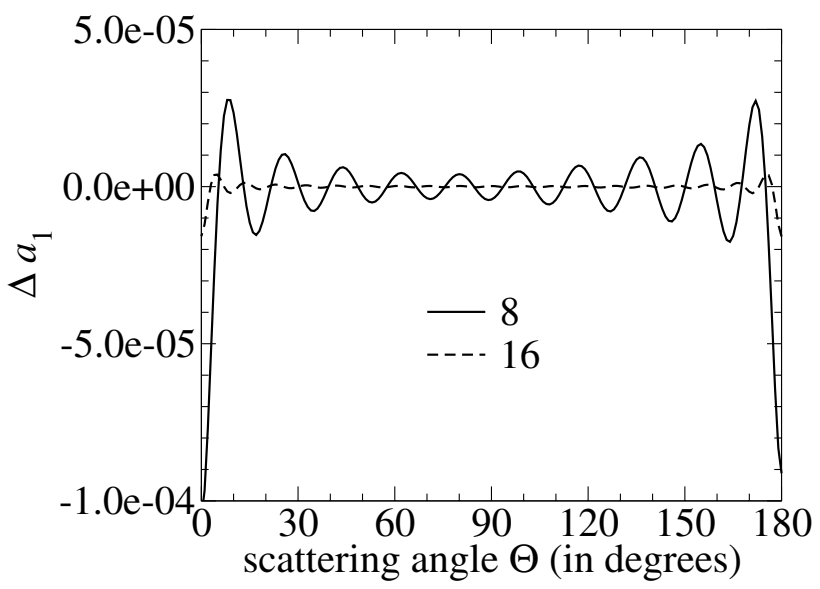

Fig. 2. The absolute error $\Delta a_{1}$ in the numerically calculated phase function $a_{1}$ of a Lambertian reflecting planet (the dashed line in Fig. 7a), if the number of Gauss-Legendre abscissae $M$ equals 8 (solid line), or 16 (dashed line). The errors are calculated as the numerically calculated values minus the analytically calculated values.

\subsection{Testing element $a_{1}$ using a Lambertian sphere}

The analytical expression for the phase function of a spherical planet with a Lambertian, perfectly reflecting surface and no atmosphere is given by (see van de Hulst 1980) (see the dashed line in Fig. 7a)

$a_{1}(\Theta)=\frac{8}{3 \pi}(\sin \Theta-\Theta \cos \Theta)$.

The geometric albedo $p$ of this planet equals 2/3 (see Eq. (81)), and since this planet reflects all incoming radiation, its Bond albedo $A_{\mathrm{B}}$ equals one (see Eq. (80)). The Fourier series expansion (see Eq. (64)) of the local reflection matrix $\boldsymbol{R}$ of a Lambertian surface has only one non-zero coefficient matrix, namely $\boldsymbol{R}^{0}$, of which the (1,1)-element equals one and all other elements equal zero. Equation (83) can thus only be used to test the accuracy of the numerical integration of $a_{1}$, and the related Bond albedo $A_{\mathrm{B}}$ and geometric albedo $p$.

Figure 2 shows the absolute errors $\Delta a_{1}$ in the numerically calculated phase function as functions of the scattering angle $\Theta$, for $M$ equal to 8 and 16 . We choose to show absolute errors rather than relative ones, because the phase function approaches zero for scattering angles close to $0^{\circ}$. The errors for $M=4,32$, 64, and 128 have been calculated but are not shown in the figure.

For $M \geq 16$, the absolute value of the error $\Delta a_{1}$ is smaller than $5 \times 10^{-6}$ across the whole scattering-angle interval, except for the forward scattering angles $\left(\Theta<2^{\circ}\right)$ and the backward scattering angles $\left(\Theta>178^{\circ}\right)$. In particular, when $\Theta=0^{\circ}$ or $180^{\circ}$, $\left|\Delta a_{1}\right|$ reaches almost $2 \times 10^{-5}$. For $M=8,\left|\Delta a_{1}\right|<5 \times 10^{-5}$ for $3^{\circ}<\Theta<177^{\circ}$, and increases up to $1 \times 10^{-4}$ for $\Theta=0^{\circ}$ and $180^{\circ}$. For $M=4,\left|\Delta a_{1}\right|<2 \times 10^{-3}$ for $10^{\circ}<\Theta<170^{\circ}$, and increases up to almost $4 \times 10^{-3}$ for $\Theta=0^{\circ}$ or $180^{\circ}$.

For the Bond albedo $A_{\mathrm{B}}$, the relative error in the numerically calculated value is smaller than $10^{-6} \%$, and thus negligible, even for $M$ as small as 4 . The relative errors in the numerically calculated geometric albedo $p$ are as small as $10^{-1} \%$ for $M=4$, about $10^{-3} \%$ for $M=8$, and as small as $10^{-4} \%$ for $M \geq 16$.

These results suggest that the accuracy of our numerical integration algorithm is in principle very high, at least for element $a_{1}$ of the scattering matrix and for the related Bond and geometric albedo.

\subsection{Testing element $b_{1}$ using single scattering}

There is no numerical reason why the integration of element $b_{1}$ (or any of the other elements) of the scattering matrix would lead to different errors than the integration of element $a_{1}$. However, the integration of element $b_{1}$ of the planetary scattering matrix $\boldsymbol{S}$ can be tested indirectly by comparing the degree of polarization $P_{\mathrm{s}}$ (Eq. (7)) of light reflected by a planet with a model atmosphere in which only single scattering takes place (assuming incident unpolarized starlight), with $P_{\mathrm{s}}$ of initially unpolarized light that is singly scattered by an ensemble of atmospheric particles (thus, without the integration over the planetary disk). For each scattering angle $\Theta$, these two cases should yield the same degree of polarization, namely $P_{\mathrm{s}}=-b_{1} / a_{1}$, with $b_{1}$ and $a_{1}$ either elements of the planetary scattering matrix or elements of the scattering matrix of the ensemble of atmospheric particles.

\subsubsection{Molecules}

First, we perform the single-scattering test with Rayleighscattering molecules, the scattering matrix of which is given in Hovenier et al. (2004). We assume a depolarization factor of 0.02 , a typical value for a planetary atmosphere that consists mainly of hydrogen, such as Jupiter's. Figure 3 shows the analytically calculated phase function $a_{1}$ and degree of polarization $P_{\mathrm{s}}$ of light singly scattered by an ensemble of molecules. The phase function is normalized such that its average over all directions equals unity. The polarization curve is symmetrical around $\Theta=90^{\circ}$, and decreases gradually towards zero in both the forward $\left(\Theta=0^{\circ}\right)$ and the backward $\left(\Theta=180^{\circ}\right)$ scattering directions.

In Fig. 4, we show the absolute errors $\Delta P_{\mathrm{s}}$ in the numerically calculated, disk-integrated $P_{\mathrm{s}}$ of a planet with a single-scattering molecular atmosphere that is bounded below by a black surface for $M=8$ and 16. Errors calculated for $M=4,32,64$, and 128 will be discussed below, but are not shown in the figure. At each value of the total scattering angle $\Theta$, the error $\Delta P_{\mathrm{s}}$ is calculated as the numerically calculated $P_{\mathrm{s}}$ minus the analytically calculated $P_{\mathrm{s}}$. As can be seen in Fig. 4, the errors in the numerically calculated $P_{\mathrm{s}}$ are generally small, but increase (in absolute sense) with decreasing $\Theta$, i.e. with the decreasing area of the disk that is illuminated and visible. For $\Theta=0^{\circ}$ and $\Theta=180^{\circ}, \Delta P_{\mathrm{s}}$ equals zero, regardless of the value of $M$.

For $M=4$ (not shown in Fig. 4), $\left|\Delta P_{\mathrm{s}}\right|<0.03$ for $\Theta>30^{\circ}$, and the maximum error (of about 0.1 ) occurs around $\Theta=15^{\circ}$. For $M=8,\left|\Delta P_{\mathrm{s}}\right|<0.001$ for $\Theta>15^{\circ}$. At scattering angles smaller than $3^{\circ},\left|\Delta P_{\mathrm{s}}\right|$ shows two sharp peaks, but remains smaller than 0.02. For $M=16,\left|\Delta P_{\mathrm{s}}\right|<0.0001$ for $\Theta>5^{\circ}$, while the largest error (0.001) occurs close to $\Theta=1^{\circ}$. For $M \geq 32$ (not shown in Fig. 4), $\left|\Delta P_{\mathrm{s}}\right|<10^{-5}$ for scattering angles larger than about $3^{\circ}$.

Our error calculations clearly show that the errors in $P_{\mathrm{s}}$ are generally small (in particularly when $M \geq 16$ ). The largest errors occur at small (but non-zero) scattering angles, when only a thin crescent of the planetary disk is illuminated and visible. Apart from the fact that at these small scattering angles our assumption of a plane-parallel model atmosphere is not valid anymore, these small angles are not even very useful for the polarimetry of extrasolar planets. Namely, at such small scattering angles, the angular distance between the extrasolar planet and its parent star will be very small, which will make it extremely difficult to spatially resolve the light reflected by the planet from the direct starlight, in particular because most of the planet's nightside is in view and thus, there is hardly any reflected light available for 


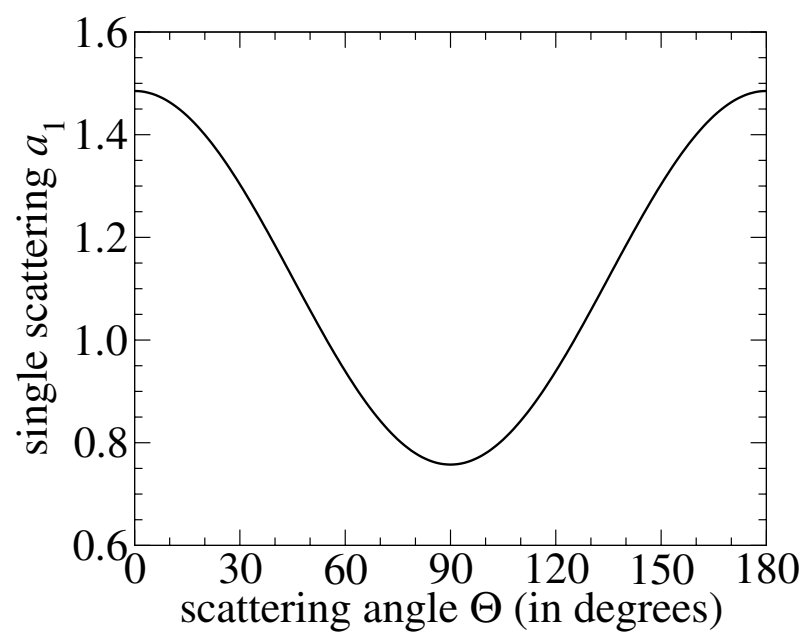

(a)

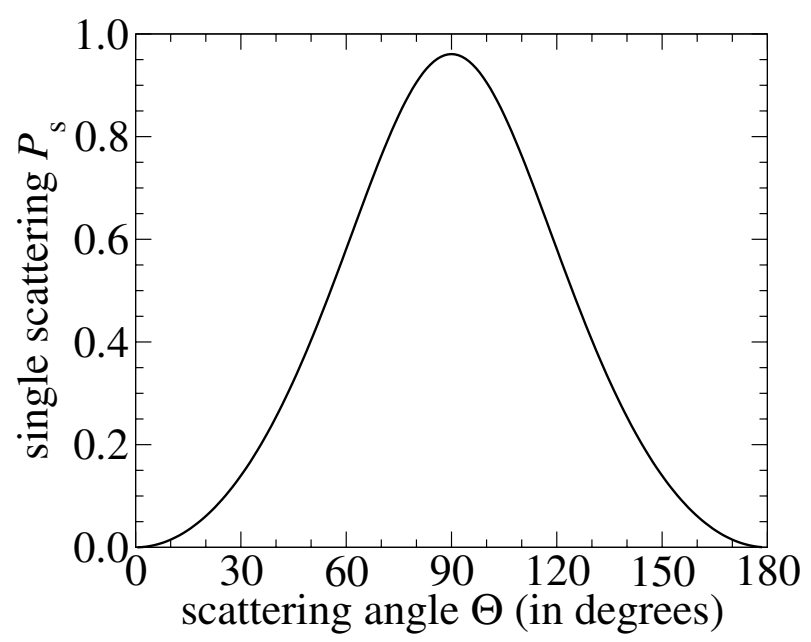

(b)

Fig. 3. a) The analytically calculated phase function $a_{1}$ of light singly scattered by an ensemble of molecules with depolarization factor 0.02 . The phase function is normalized such that its average over all directions equals unity. b) The analytically calculated degree of polarization $P_{\mathrm{s}}=-b_{1} / a_{1}$ of this scattered light.

observations. Furthermore, at these angles, the degree of polarization of the light reflected by the planet is very close to zero, making polarimetry very difficult.

Concluding, our numerical integration algorithm is an accurate tool for calculating $P_{\mathrm{s}}$ and $b_{1}$ at least when the elements $a_{1}$ and $b_{1}$ of the scattering matrix of the atmospheric particles are smooth functions of the single scattering angle, i.e. without sharp angular features.

\subsubsection{Aerosol particles}

Because the matrix elements describing Rayleigh scattering are smooth functions of the scattering angle, we performed a second single scattering test using atmospheric aerosol particles that were chosen especially for the sharp angular features in their scattering matrix elements, namely the model D particles that are presented by de Rooij \& van der Stap (1984) with their optical properties at $\lambda=0.70 \mu \mathrm{m}$. We preferred using a wavelength of $0.55 \mu \mathrm{m}$ (to keep in line with our other work). Like de Rooij $\&$ van der Stap (1984), we used a refractive index of 1.33; the single scattering albedo of the aerosol particles is thus 1.0. The

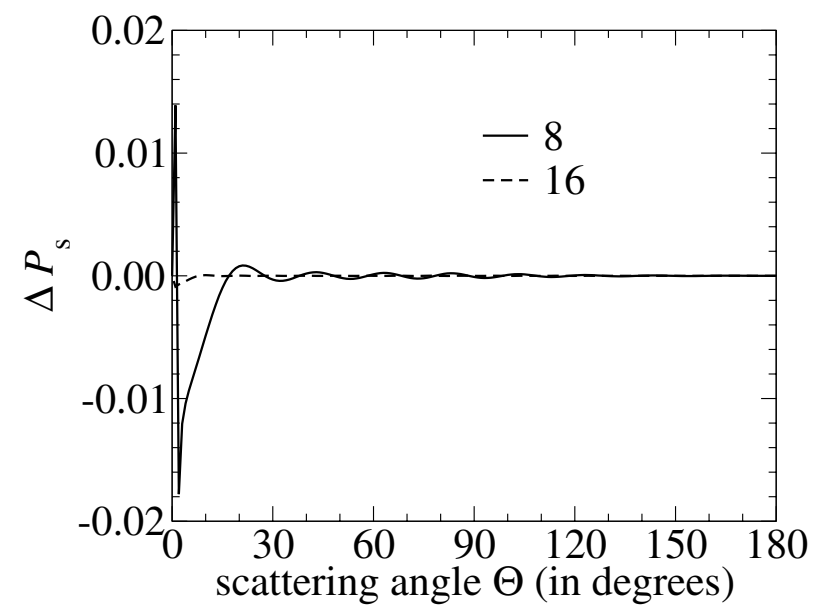

Fig. 4. The absolute errors $\Delta P_{\mathrm{s}}$ in the numerically calculated, diskintegrated $P_{\mathrm{s}}$ of a planet with a single scattering molecular atmosphere bounded below by a black surface as functions of the total scattering angle $\Theta$, for $M=8$ (solid line) and $M=16$ (dashed line). The errors are calculated as the numerically calculated values minus the analytically calculated values.

scattering matrix elements of the model D particles were calculated using Mie theory (van de Hulst 1957). Figure 5 shows the analytically calculated phase function $a_{1}$ and degree of polarization $P_{\mathrm{s}}$ of light singly scattered by an ensemble of model D particles at $\lambda=0.55 \mu \mathrm{m}$. Note that the feature at scattering angles slightly smaller than $150^{\circ}$ in both $a_{1}$ and $P_{\mathrm{s}}$ is the primary rainbow. The feature at scattering angles slightly smaller than $180^{\circ}$ is generally referred to as the glory.

In Fig. 6, we show the absolute errors $\Delta P_{\mathrm{s}}$ in the numerically calculated, disk-integrated $P_{\mathrm{s}}$ of a planet with a singlescattering atmosphere that contains only model $\mathrm{D}$ particles and that is bounded below by a black surface, for $M=8,16$, and 32 . Errors calculated for $M=4,64$, and 128 will be discussed below, but are not shown in the figure. Interestingly, for all values of $M$, the largest (in absolute sense) errors in this case appear not at small but at large scattering angles $\left(\Theta>175^{\circ}\right)$ (the slight increase in the errors with decreasing $\Theta$ at the smallest scattering angles still occurs but is negligible compared to the errors at the largest scattering angles). The errors at the largest scattering angles are due to the sharp peaks in $P_{\mathrm{s}}$ at these angles (see Fig. 5b). Note that at $\Theta=0^{\circ}$ and $180^{\circ}$, the errors vanish for all values of $M$.

Using the model D particles and $M=4$ (not shown in Fig. 6), the error $\Delta P_{\mathrm{s}}$ shows a similar scattering angle dependence as the analytically calculated $P_{\mathrm{s}}$ (Fig. 5b), with a maximum error of about 0.5 around $\Theta=160^{\circ}$. For $M=8$, the largest errors are on the order of 0.1 . The sharp peak in $\left|\Delta P_{\mathrm{s}}\right|$ up to 0.2 at $\Theta=177^{\circ}$ coincides with the sharp peak in the analytically calculated $P_{\mathrm{s}}$ (Fig. 5b). For $M=16,\left|\Delta P_{\mathrm{s}}\right|<0.01$ for $\Theta<166^{\circ}$. As for $M=8$, the largest error (of 0.25 ) occurs at $\Theta=177^{\circ}$. For $M=32$, $\left|\Delta P_{\mathrm{s}}\right|$ is generally much smaller than 0.005 , except at the largest scattering angles, where an error of almost 0.04 occurs (near $\Theta=$ $177^{\circ}$ ). With $M=64$ (not shown in Fig. 6), the sharp peak at $\Theta=$ $177^{\circ}$ is almost resolved, so the largest errors in this scattering angle region are reduced to below 0.005 . With $M=128$, the largest errors in $\left|\Delta P_{\mathrm{s}}\right|$ are even smaller than 0.0005 .

Apparently, for the planetary atmosphere with model D particles, the errors at the largest scattering angles are generally larger than those at the smaller scattering angles, for each value of $M$. This is most probably due to the angular features in $P_{\mathrm{s}}$ at the largest scattering angles (cf. Fig. 5b). The errors at the 


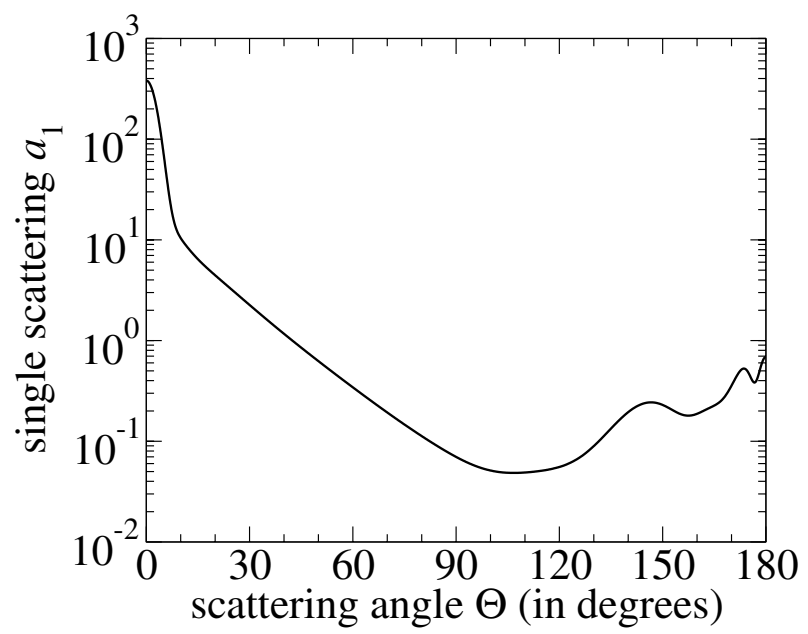

(a)

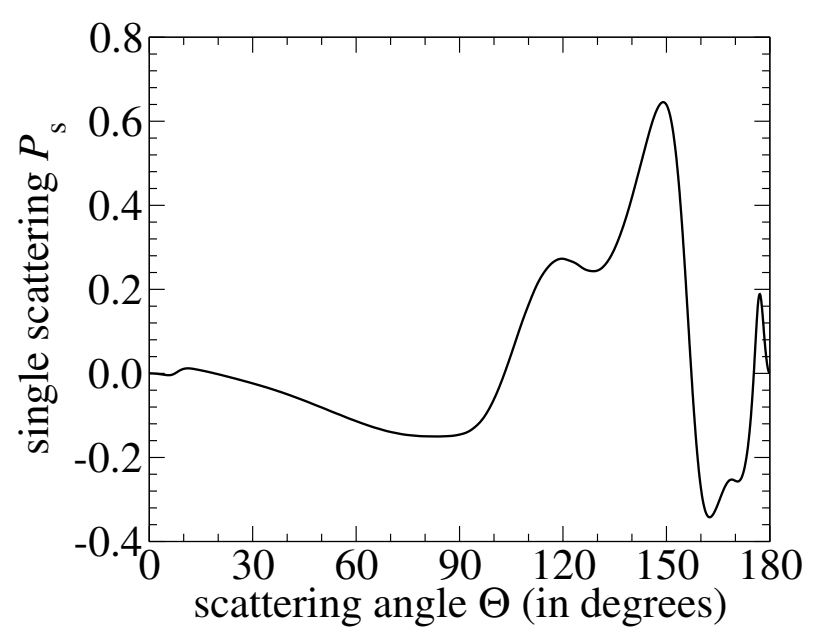

(b)

Fig. 5. a) The analytically calculated phase function $a_{1}$ of light singly scattered by an ensemble of model D particles (de Rooij \& van der Stap 1984) at $\lambda=0.55 \mu \mathrm{m}$. The phase function is normalized such that its average over all directions equals unity. b) The analytically calculated degree of polarization $P_{\mathrm{s}}=-b_{1} / a_{1}$ of this scattered light.

largest scattering angles are still sufficiently small to safely conclude that our numerical integration algorithm also works well for calculating $P_{\mathrm{s}}$ when it has very sharp angular features.

\section{Sample integrations}

In Sect. 3, we have tested our numerical disk-integration algorithm by comparing its results with results obtained using other algorithms. The model planets used in Sect. 3 represent special cases, such as having a single-scattering atmosphere. In this section, we present properties of the disk-integrated light that is reflected by two model planets with more realistic, multiple scattering atmospheres. We compare scattering matrix elements $a_{1}$ (the phase function) and $b_{1}$, degrees of polarization, and Bond and geometric albedos for different values of the number of Gaussian abscissae $M$.

\subsection{A molecular model atmosphere}

Figure 7 shows for $\lambda=0.55 \mu \mathrm{m}$ and unpolarized incident light the phase function $a_{1}$ and the degree of polarization $P_{\mathrm{s}}$

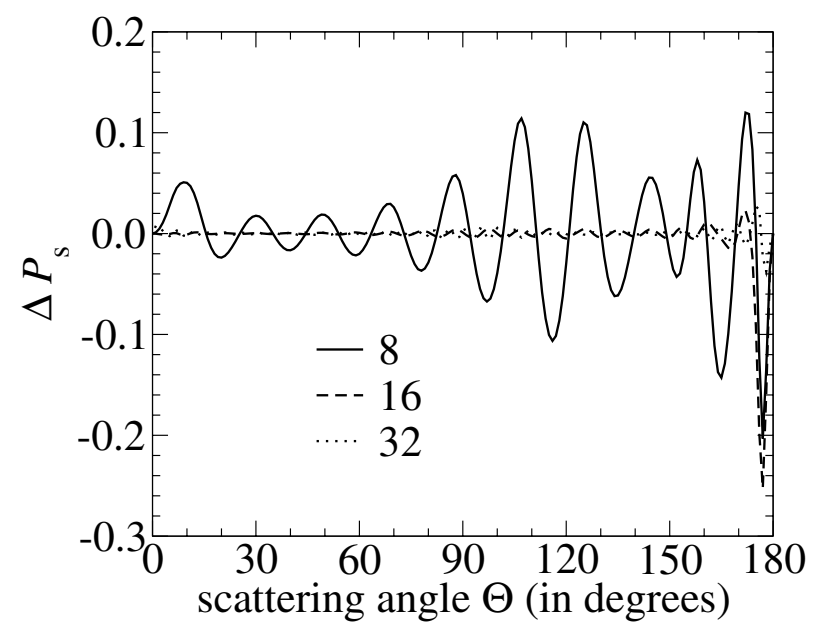

Fig. 6. The absolute errors $\Delta P_{\mathrm{s}}$ in the numerically calculated, diskintegrated $P_{\mathrm{s}}$ of a planet with a single scattering atmosphere that contains only model D aerosol and that is bounded below by a black surface as functions of the total scattering angle $\Theta$, for $M=8$ (solid line), 16 (dashed line), and 32 (dotted line). The wavelength $\lambda=0.55 \mu \mathrm{m}$. The errors are calculated as the numerically calculated values minus the analytically calculated values.

as functions of the scattering angle $\Theta$ for a model atmosphere that contains only molecules and that is bounded below by a black surface. The atmospheric absorption optical thickness is chosen equal to zero, and the molecular depolarization factor is 0.02 (Hansen \& Travis 1974). The atmospheric scattering optical thickness is 5.75 (at $0.55 \mu \mathrm{m}$ ). This optical thickness is representative of a Jupiter-like atmosphere down to a pressure of 5.6 bars. At $0.55 \mu \mathrm{m}$, the geometric albedo $p$ of this planet is 0.6471 (i.e. $\frac{1}{4} a_{1}\left(180^{\circ}\right)$ ) and its Bond albedo is 0.8141 . The number of Gaussian abscissae $M$ used for these calculations is 128 . Calculations with a larger number of abscissae did not have a noticeble effect on the results, so that we can use the results for $M=128$ as a reference when employing lower values of $M$.

For comparison in Fig. 7a, we have also plotted the phase function of a Lambertian reflecting planet (Eq. (83)), scaled to the geometric albedo of the planet with the molecular atmosphere (i.e. 0.6471). Clearly, the model planet with the Jupiterlike molecular atmosphere is darker for $60^{\circ}<\Theta<180^{\circ}$ (or $0^{\circ}<\alpha<120^{\circ}$ ) than a Lambertian reflecting planet with the same geometric albedo.

There are a number of differences between the degree of polarization $P_{\mathrm{s}}$ of this planet with a multiple-scattering molecular atmosphere (Fig. 7b) and that of light singly scattered by molecules (Fig. 3b). Firstly, because of the multiple scattering, the maximum degree of polarization $P_{\mathrm{s}}$ of the light reflected by this planet is much lower than that of the singly-scattered light, namely 0.37 versus 0.96 . Secondly, for the planet, the scattering angle at which $P_{\mathrm{s}}$ achieves its maximum value is $87^{\circ}$, versus $90^{\circ}$ for the singly-scattered light. Thirdly, at scattering angles smaller than $17.6^{\circ}$, when only a crescent of the planet is visible, $P_{\mathrm{s}}$ of the planet is negative (thus, the light is polarized parallel to the reference plane through the planet, the observer, and the star), whereas $P_{\mathrm{s}}$ of the singly scattered light remains positive. The negative polarization at small scattering angles is mainly due to second-order scattered light (see van de Hulst 1980). The dashed line in Fig. 7b shows $P_{\mathrm{s}}$ as expected for a Lambertian reflecting planet. Obviously, because the reflection by a Lambertian reflecting planet is unpolarized, $P_{\mathrm{s}}=0$ independent of $\Theta$. 


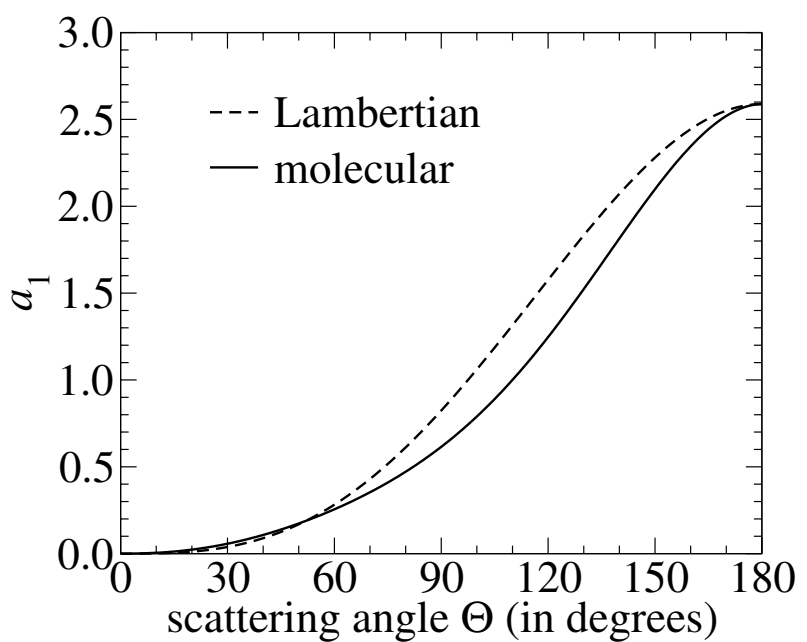

(a)

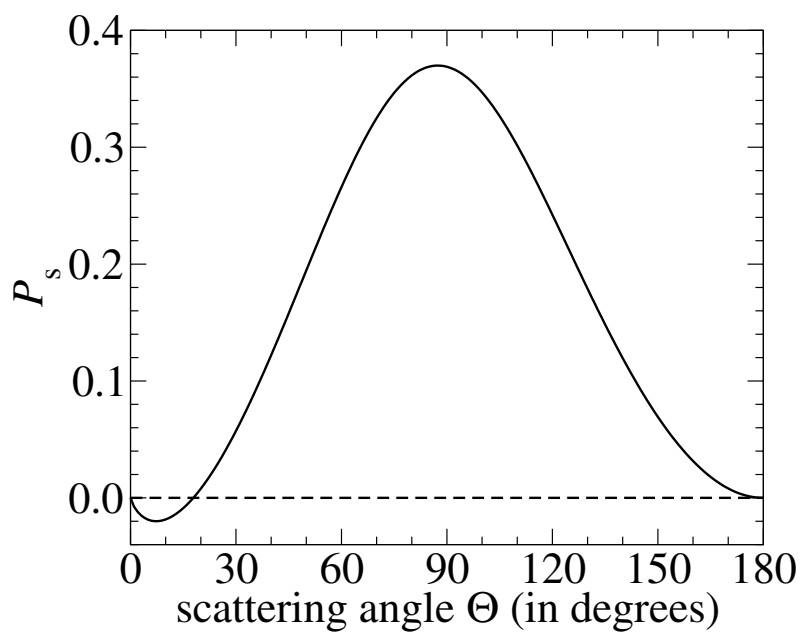

(b)

Fig. 7. a) The numerically calculated $(M=128)$ phase function $a_{1}$ for a planet with a purely molecular model atmosphere bounded below by a black surface with an optical thickness of 5.75 (solid line) and the analytically calculated phase function of a Lambertian reflecting planet (dashed line), and $\mathbf{b})$ the degree of polarization $P_{\mathrm{s}}$ for the planet with the molecular model atmosphere and the Lambertian planet, all as functions of the total scattering angle $\Theta$.

In Fig. 8, we have plotted the absolute errors $\Delta a_{1}$ and $\Delta P_{\mathrm{s}}$ when the planetary scattering matrix elements calculated for $M=128$ are compared to those calculated for $M=8,16$, and 32. The errors for $M=4$ and $M=64$ have been calculated and will be discussed, but are not shown in the figure. Similarly, the errors in scattering matrix element $b_{1}$ have not been plotted, but will be discussed.

For a planet with a molecular model atmosphere, the error $\left|\Delta a_{1}\right|$ in the phase function $a_{1}$ (Fig. 8a) due to using less than 128 Gaussian abscissae, is on the order of 0.005 when $M=4$ for all scattering angles (not shown in the figure), generally smaller than $10^{-4}$ when $M=8$, except when $\Theta<4^{\circ}\left(\Delta a_{1}=0.00026\right.$ when $\left.\Theta=0^{\circ}\right)$, and when $\Theta>167^{\circ}\left(\Delta a_{1}=0.0013\right.$ when $\Theta=180^{\circ}$ ). For $M=16,\left|\Delta a_{1}\right|<10^{-5}$ across most of the scattering angle interval; for $\Theta<2^{\circ}$ the error is somewhat larger, in particular, when $\Theta=0^{\circ}, \Delta a_{1}=2 \times 10^{-5}$. For $M=16$ and $\Theta>165^{\circ}$, the errors increase up to 0.00028 when $\Theta=180^{\circ}$. For $M=32$ and $3^{\circ}<\Theta<160^{\circ},\left|\Delta a_{1}\right|<5 \times 10^{-7}$. When $\Theta=0^{\circ}$, $\Delta a_{1}=2.5 \times 10^{-6}$, and when $\Theta=180^{\circ}, \Delta a_{1}=6 \times 10^{-5}$. Finally,

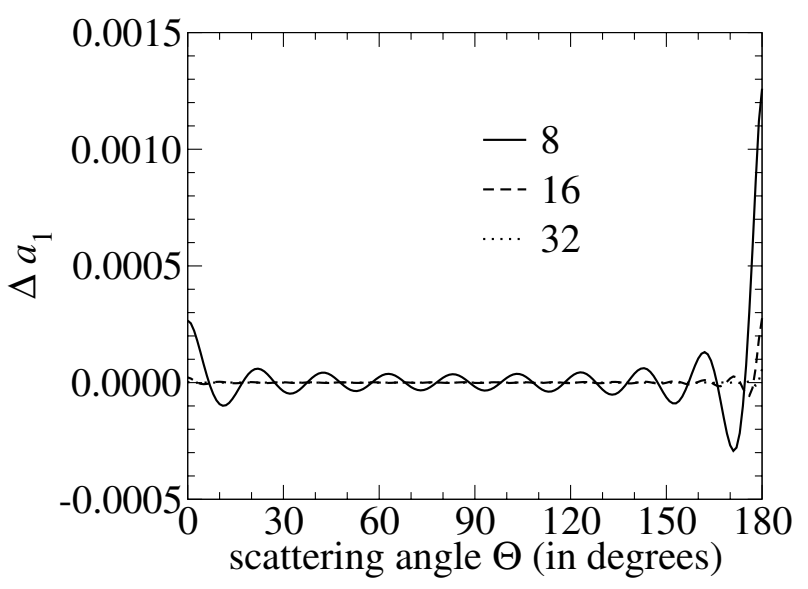

(a)

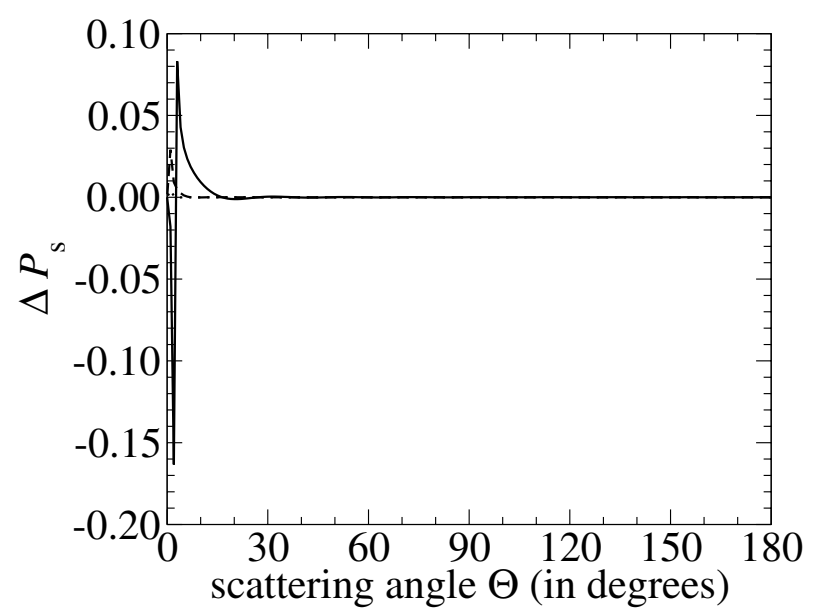

(b)

Fig. 8. The absolute errors on the curves in Fig. 7 when $M=8$ (solid line), 16 (dashed line), or 32 (dotted line), instead of 128 in a) the phase function $a_{1}$, and $\left.\mathbf{b}\right)$ the degree of polarization $P_{\mathrm{s}}$. The errors are calculated as the results for $M=128$ minus the results at another value of $M$.

when $M=64$ (not shown in Fig. 8a), $\left|\Delta a_{1}\right|<10^{-7}$, except when $\Theta>177^{\circ}$; in particular, when $\Theta=180^{\circ}, \Delta a_{1}=10^{-5}$.

The errors in scattering matrix element $b_{1}$ (not shown in a figure), are about $50 \%$ smaller than those in element $a_{1}$, and they show a similar scattering-angle dependence. A difference is that the errors in $b_{1}$ vanish at $\Theta=0^{\circ}$ and at $\Theta=180^{\circ}$ regardless of the value of $M$. At these two scattering angles, $b_{1}$ itself equals zero, too.

The errors in $P_{\mathrm{s}}$ (Fig. 8b) are generally largest at small scattering angles, except at $\Theta=0^{\circ}$, where all errors vanish regardless of the value of $M$, like those in $b_{1}$. For $M=4$ (not shown in Fig. 8b), the largest $\Delta P_{\mathrm{s}}$ is 0.15 , which occurs near $\Theta=15^{\circ}$. When $\Theta>50^{\circ},\left|\Delta P_{\mathrm{s}}\right|<0.01$. For $M=8,\left|\Delta P_{\mathrm{s}}\right|<0.001$ when $\Theta>20^{\circ}$, and $\left|\Delta P_{\mathrm{s}}\right|<0.0001$ when $\Theta>40^{\circ}$. Note that the two sharp peaks in $\Delta P_{\mathrm{S}}$ occur in the scattering angle range where $P_{\mathrm{S}}<0$. For $M=16,\left|\Delta P_{\mathrm{s}}\right|$ has a sharp peak equal to 0.03 around $\Theta=1^{\circ}$, and decreases rapidly with increasing $\Theta$; when $\Theta>10^{\circ}$, $\left|\Delta P_{\mathrm{s}}\right|<5 \times 10^{-5}$. A similar rapid decrease of $\left|\Delta P_{\mathrm{s}}\right|$ with $\Theta$ is seen for $M=32$; there is a sharp peak equal to 0.004 around $\Theta=1^{\circ}$, and when $\Theta>30^{\circ},\left|\Delta P_{\mathrm{s}}\right|<2 \times 10^{-7}$. For $M=64$, finally, the maximum error is about $4 \times 10^{-4}$ (around $\Theta=1^{\circ}$ ), and when $\Theta>10^{\circ},\left|\Delta P_{\mathrm{s}}\right|<4 \times 10^{-8}$. Thus, as seen before, when one requires simulations for scattering angles equal or close to $0^{\circ}$, a larger number of Gaussian abscissae should be used for the 
calculation of $a_{1}, b_{1}$, and $P_{\mathrm{s}}$, than when one is only interested in simulations for larger scattering angles (i.e. smaller phase angles).

The relative errors in the numerical simulations of the Bond and geometric albedo are small when comparing albedos calculated using $M<128$ to those calculated using $M=128$. The relative error in the geometric albedo $p$ is smaller than $0.05 \%$ for $M$ as small as 8 . The calculations of the Bond albedo are even more accurate: with 8 abscissae, the relative error is only $2.5 \times 10^{-6} \%$.

\subsection{A model atmosphere with aerosol particles}

We performed similar calculations to those in Sect. 4.1 with aerosol particles added to the molecular model atmosphere. For the aerosol particles, we chose, as in Sect. 3.2.2, the model D particles described by de Rooij \& van der Stap (1984) because of the sharp features in their scattering matrix elements (see Fig. 5). To enhance the influence of the scattering matrix of the aerosol particles, a large aerosol optical thickness is chosen, namely 3.25 , yielding a total atmospheric optical thickness of 9.0 (at $\lambda=0.55 \mu \mathrm{m}$ ). The aerosol particles are well-mixed throughout the atmosphere; i.e. the atmosphere is homogeneous. We will refer to this model atmosphere with aerosol particles and bounded below by a black surface, as the aerosol atmosphere.

Figure 9 shows $a_{1}$ and $P_{\mathrm{s}}$ as functions of $\Theta$ at $\lambda=0.55 \mu \mathrm{m}$ for the aerosol atmosphere. For comparison, this figure also contains the curves for the purely molecular model atmosphere (see Sect. 4.1). The geometric albedo of the planet with the aerosol atmosphere is 0.6688 , and its Bond albedo is 0.8281 (at $\lambda=0.55 \mu \mathrm{m})$. For these calculations, we used $M=128$.

As can be seen in Fig. 9a, adding the model D particles to the molecular model atmosphere hardly changes the planetary phase function even though 1) the single-scattering phase function of the particles differs significantly from the molecular single scattering phase function (compare Figs. 3a and 5a), and 2 ) the aerosol optical thickness is relatively large. The largest difference between the two phase functions shown in Fig. 9a is in the backward scattering direction, for $\Theta>160^{\circ}$. The angular feature in the phase function of the planet with the aerosol atmosphere that shows up at these scattering angles can be traced back to a feature in the particles' single-scattering phase function (see Fig. 5a).

Figure $9 \mathrm{~b}$ shows that the scattering angle dependence of $P_{\mathrm{s}}$ for the planet with the aerosol atmosphere differs significantly from that of the planet with the purely molecular atmosphere. Not only is $P_{\mathrm{s}}$ for the aerosol atmosphere lower than for the molecular atmosphere across most of the scattering-angle interval $\left(20^{\circ}<\Theta<170^{\circ}\right)$, but it also shows some angular features that are related to features in the aerosol's single scattering degree of polarization (see Fig. 5).

In Fig. 10, we have plotted the absolute errors in $a_{1}$ and $P_{\mathrm{s}}$ when instead of $M=128$, we use $M=16,32$, or 64 for the aerosol atmosphere. Results for matrix element $b_{1}$ and for $M=8$ are not shown, but will be discussed. When only 4 Gaussian abscissae were used for this model atmosphere loaded with model $\mathrm{D}$ aerosol particles, our adding-doubling algorithm did not converge very well, so in this section, we ignore $M=4$.

For the planet with the aerosol atmosphere, the error $\left|\Delta a_{1}\right|$ in the phase function $a_{1}$ is smaller than 0.03 for $M=8$ (not shown in Fig. 10a) when $\Theta<140^{\circ}$. At the largest scattering angles, $\left|\Delta a_{1}\right|$ reaches a maximum of about 0.15 at $\Theta=177^{\circ}$ for $M=8$. This large error is due to the sharp angular feature exhibited in the single scattering by model D aerosol particles (see Fig. 5).

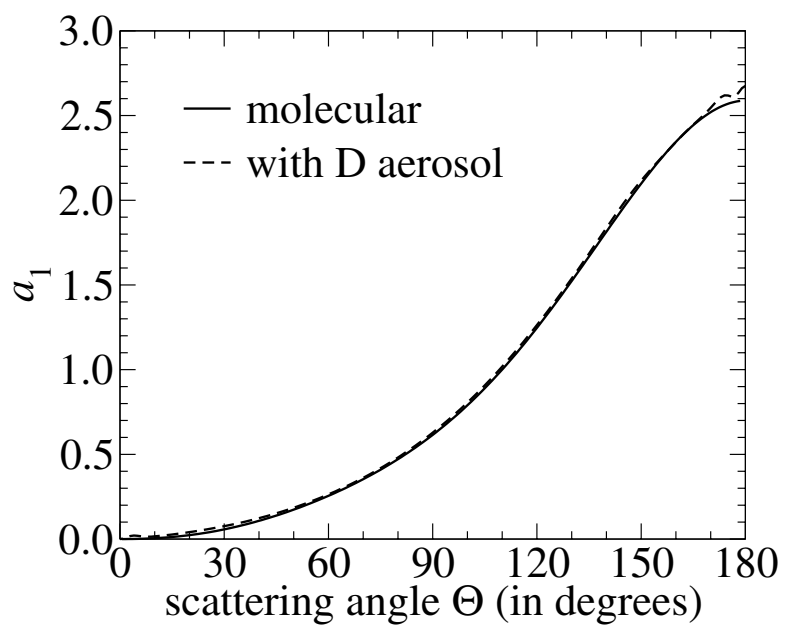

(a)

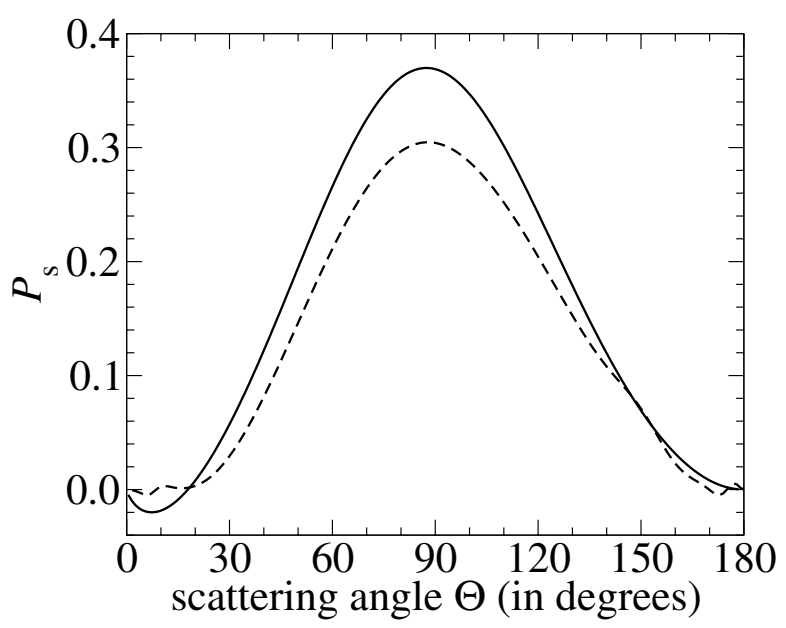

(b)

Fig. 9. The numerically calculated a) phase function $a_{1}$, and b) degree of polarization $P_{\mathrm{s}}$ for a planet with a molecular atmosphere containing model D aerosol (de Rooij \& van der Stap 1984), as functions of the total scattering angle $\Theta$, for $M=128$ and $\lambda=0.55 \mu \mathrm{m}$ (dashed lines). The total optical thickness of this aerosol atmosphere is 9.0 (at a wavelength of $0.55 \mu \mathrm{m}$ ). For comparison, the curves for a purely molecular model atmosphere (with optical thickness 5.75) (see Fig. 7) have also been included (solid lines). Both atmospheres are bounded below by a black surface.

For $M=16, \Delta a_{1}$ shows a strong angular dependence, reflecting angular features in the single scattering phase function of the model D particles (see Fig. 5a). For $6^{\circ}<\Theta<140^{\circ}$, the errors remain smaller than 0.002 (in absolute sense). The largest error, i.e. of 0.03 , occurs at $\Theta=177^{\circ}$, and is related to the sharp angular feature in the single scattering by model $\mathrm{D}$ aerosol particles (see Fig. 5). For $M=32$, the errors in $\left|a_{1}\right|$ are smaller than $10^{-4}$ when $\Theta<140^{\circ}$. With an increasing scattering angle up from $140^{\circ},\left|\Delta a_{1}\right|$ increases up to 0.013 at $\Theta=180^{\circ}$. For $M=64$, the errors in $\left|a_{1}\right|$ are smaller than $5 \times 10^{-5}$, except for $\Theta>150^{\circ}$. The error $\left|\Delta a_{1}\right|$ is largest at $\Theta=180^{\circ}$, i.e. 0.003 .

The errors in scattering-matrix element $b_{1}$ are similar to those in $a_{1}$, not only in value but also in their angular dependence. The only difference is that the errors in $b_{1}$ converge to zero at $\Theta=0^{\circ}$ and $\Theta=180^{\circ}$, where $b_{1}$ itself equals zero.

Figure $10 \mathrm{~b}$ shows the absolute errors in $P_{\mathrm{s}}$. For all values of $M, \Delta P_{\mathrm{s}}$ equals zero at $\Theta=0^{\circ}$ and $\Theta=180^{\circ}$. For $M=8$ (not shown in Fig. 10b), $\left|\Delta P_{\mathrm{s}}\right|<0.05$ at most scattering angles, except for intermediate scattering angles, i.e. $50^{\circ}<\Theta<130^{\circ}$. 


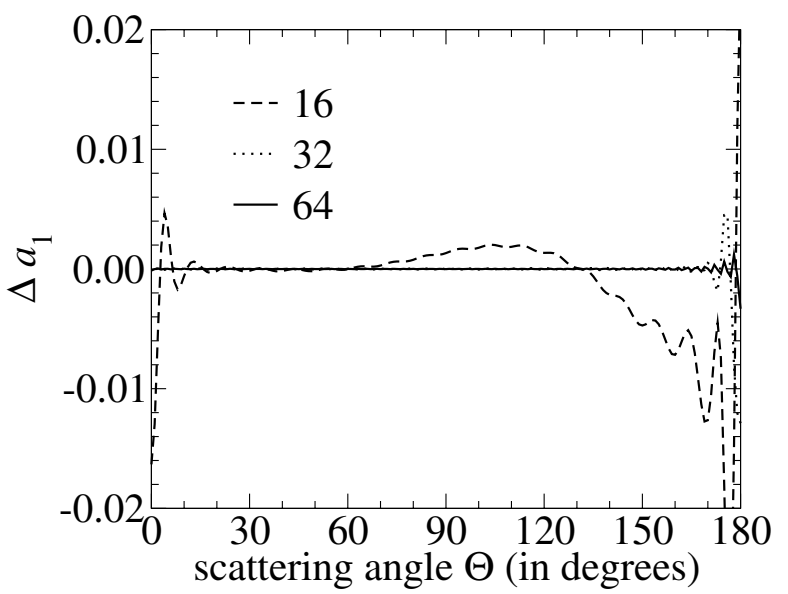

(a)

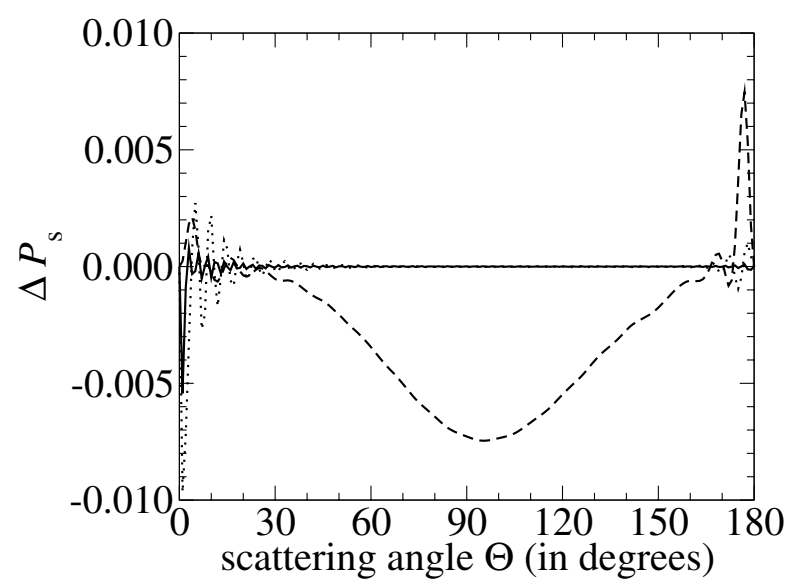

(b)

Fig. 10. The absolute errors in a) the phase function $a_{1}$, and b) the degree of polarization $P_{\mathrm{s}}$ of the planet with the same aerosol atmosphere as in Fig. 9, when using $M=16,32$, or 64, instead of 128. The errors are calculated as the results for $M=128$ minus the results at another value of $M$.

Around $\Theta=90^{\circ},\left|\Delta P_{\mathrm{s}}\right|$ reaches a maximum value of 0.10 . The location of this maximum error coincides with a local minimum in $P_{\mathrm{s}}$ of the singly-scattered light (see Fig. 5b). For $M=16,\left|\Delta P_{\mathrm{s}}\right|$ is smaller than 0.008 for all scattering angles. For $M=32,\left|\Delta P_{\mathrm{s}}\right|$ is smaller than $10^{-4}$ for $40^{\circ}<\Theta<170^{\circ}$. With $\Theta$ decreasing from $40^{\circ},\left|\Delta P_{\mathrm{s}}\right|$ increases to reach a sharply-peaked maximum value of 0.01 at $\Theta=1^{\circ}$. With $\Theta$ increasing from $170^{\circ},\left|\Delta P_{\mathrm{s}}\right|$ increases, but remains smaller than 0.001 . For $M=64,\left|\Delta P_{\mathrm{s}}\right|$ is smaller than $5 \times 10^{-5}$ for $30^{\circ}<\Theta<170^{\circ}$. For scattering angles smaller than $30^{\circ},\left|\Delta P_{\mathrm{s}}\right|$ reaches a maximum value of 0.005 (at $\left.\Theta=1^{\circ}\right)$. For scattering angles larger than $170^{\circ},\left|\Delta P_{\mathrm{s}}\right|$ reaches a maximum value of 0.00015 (near $\Theta=178^{\circ}$ ).

As for the case with the purely molecular model atmosphere (Sect. 4.1), the numerical simulations of the Bond albedo $A_{\mathrm{B}}$ and the geometric albedo $p$ are quite insensitive to the number of Gaussian abscissae. Compared to results for $M=128$, the relative error in $p$ is smaller than $1 \%$ for $M=16$, and about $4 \%$ for $M$ as small as 8 . The relative error in $A_{\mathrm{B}}$ is smaller than $0.01 \%$ with as little as 8 Gaussian abscissae.

\section{Summary and discussion}

In the previous sections, we have described and tested our diskintegration method for light reflected by a spherical planet. The method is based on the expansion of the radiation field of a horizontally homogeneous planet into generalized spherical functions. Our tests for the planetary phase function $a_{1}, b_{1}$, and the degree of linear polarization $P_{\mathrm{s}}$ of the reflected light (assuming unpolarized incoming light) show that for a given planetary model atmosphere, the accuracy of the method depends strongly on 1) the number $M$ of Gaussian abscissae that are used in the integration of the Fourier coefficients of the planetary reflection matrix, and 2) the scattering angle $\Theta$ (i.e. $180^{\circ}-\alpha$, with $\alpha$ the planetary phase angle).

We calculated the errors of our disk-integration method for two planetary model atmospheres: one containing only gaseous molecules, and the other containing gases and model D aerosol particles (see de Rooij \& van der Stap 1984). We chose these model $\mathrm{D}$ aerosol particles, because light that is scattered by these particles shows very strong angular features, both in intensity and polarization (see Fig. 5). The model atmosphere containing these particles thus provides an extreme case for our diskintegration algorithm.

We found that for a model planet with an atmosphere containing only gaseous molecules, the absolute error $\left|\Delta a_{1}\right|$ in the planetary phase function $a_{1}$, is smaller than $10^{-5}$ across most of the scattering interval for $M \geq 16$. The absolute errors $\left|\Delta P_{\mathrm{s}}\right|$ in $P_{\mathrm{s}}$, are smaller than $5 \times 10^{-5}$ for $M \geq 16$ (except at scattering angles smaller than about $10^{\circ}$. For the model atmosphere containing both molecular gases and model D aerosol particles (with optical thicknesses of, respectively, 5.75 and 3.25 at $0.55 \mu \mathrm{m}), M$ should be larger than 32 to keep the absolute errors in the disk-integrated $a_{1}$ and $P_{\mathrm{s}}$ smaller than $10^{-4}$. Note that light that has been singly scattered by model $\mathrm{D}$ aerosol particles shows sharp angular features in both the intensity and the degree of polarization. These angular features are the main source of errors for the disk-integration algorithm, simply because the number of Gaussian abscissae should be chosen large enough to resolve the angular features. In general, the elements of the single-scattering matrix of aerosol particles will be much smoother functions of the scattering angle than those of the model D particles. Obviously, when the elements of the atmospheric single-scattering matrix (which is a weighted sum of the scattering matrices of the various atmospheric constituents) are smooth functions of the scattering angle, lower values of $M$, e.g. 16 , will be sufficient. Scattering matrix elements that are smooth functions of the scattering angle can be expected e.g. when the aerosol particles are small compared to the wavelength, when these particles have irregular shapes (see Volten et al. 2005), or when there are few aerosol particles compared to gaseous molecules.

The errors in the disk-integrated $a_{1}, b_{1}$, and $P_{\mathrm{s}}$ depend, for a given value of $M$, on the scattering angle $\Theta$. Generally, the angular variation of the errors depends strongly on the angular dependence of the single-scattering phase function of the particles in the planetary atmosphere: at scattering angles where this phase function shows sharp features, the errors of the integration algorithm will be relatively large for a given number $M$. This relation between the angular features in the single-scattering phase function of the atmospheric particles and the number of geometries or locations on the planet required for integrating reflected light over the planetary disk will probably hold for any diskintegration algorithm.

These types of errors in the disk-integrated $a_{1}, b_{1}$, and $P_{\mathrm{s}}$ usually occur at the largest scattering angles (when most of the planet's dayside is in view), because at these geometries, narrow angle features in the single-scattering phase functions of spherical atmospheric particles, in particular, such as haze or cloud 
patricles are quite common (see Fig. 5, and examples in Hansen $\&$ Travis 1974). Relatively large errors in $a_{1}$ can also be found at the smallest scattering angles, where only a narrow crescent of the planet is illuminated and in view. The errors in $b_{1}$ are similar to those in $a_{1}$, except that they equal zero at $\Theta=0^{\circ}$ and $\Theta=180^{\circ}$, where $b_{1}$ itself equals zero.

The relatively large errors in $a_{1}$ that occur at the smallest scattering angles also show up in the absolute error in $P_{\mathrm{s}}$, simply because $P_{\mathrm{s}}=-b_{1} / a_{1}$. At $\Theta=0^{\circ}$ and $\Theta=180^{\circ}$, however, the errors in $P_{\mathrm{s}}$ are zero, like those in $b_{1}$ (assuming unpolarized incident light). The error in $P_{\mathrm{s}}$ is, like that in $a_{1}$ and $b_{1}$, also sensitive to the scattering-angle dependence of the scattering functions of the atmospheric constituents: the sharper the angular features in the single scattering, the larger the errors that can be expected at those angles in the disk-integrated $P_{\mathrm{s}}$.

For a given value of $M$, the largest errors in the diskintegrated $a_{1}, b_{1}$, and $P_{\mathrm{s}}$ are thus generally found at the smallest and largest scattering angles. In practise, it will not be necessary to use extra high values of $M$ to minimize such errors, because the smallest and largest scattering angles are not very useful for direct observations of extrasolar planets. Namely, at such scattering angles, the angular distance between the extrasolar planet and its parent star is very small, which will make it extremely difficult to spatially resolve the light reflected by the planet from the direct, unscattered starlight. Even if no attempt is made for spatially resolving the planet, for example because the polarized signal of the planet will be searched for in the unpolarized signal of the star (Seager et al. 2000; Hough \& Lucas 2003), these scattering angles are of little use, because $P_{\mathrm{s}}$ can be expected to be close to zero (see Figs. $7 b$ and $9 b$ ).

The errors resulting from applying our disk-integration algorithm to a specific model planet usually decrease with an increasing number of Gaussian abscissae $M$. The time required for computing the disk-integrated flux vector of the light reflected by the model planet is mainly spent in two numerical routines, namely one for calculating the Fourier coefficients $\boldsymbol{R}^{s}$ of the local reflection matrix and one for integrating these coefficients over the planetary disk (and hence obtaining the planetary reflection matrix $\boldsymbol{S}$ ). It appears that the computing time required for the diskintegration is negligible compared to what is required for calculating the reflection function of a plane-parallel atmosphere, in particular for $M \geq 32$. The time required for computing diskintegrated flux vectors will thus mainly depend on the efficiency of the radiative transfer calculations of the user.

We use an efficient adding-doubling algorithm (de Haan et al. 1987) for the radiative transfer calculations. With our computer code (in Fortran'77), the time required for computing the Fourier coefficients increases strongly with the number of Gaussian abscissae $M$ (see de Haan et al. 1987). When planning large numbers of calculations of disk-integrated flux vectors, e.g. for the interpretation of observations, it is obviously advantageous to keep $M$ as small as possible. A method to minimize both $M$ and the errors in the disk-integrated $a_{1}$ and $P_{\mathrm{s}}$, would be interpolation between Fourier coefficients of the local reflection matrix $\boldsymbol{R}$ as follows. The Fourier coefficients $\boldsymbol{R}^{s}$ are calculated at an $N \times N$ grid of $\left(\mu, \mu_{0}\right)$-pairs, and before integrating these Fourier coefficients over the planetary disk, they are interpolated onto an $M \times M$ grid (with $M>N$ ). Test calculations we performed show that this method works very well across most of the scattering-angle region, i.e. $N$ can be as small as $\frac{1}{2} M$ without a significant increase in the errors. The method works less well for scattering angles where errors are due to sharp angular features in the single-scattering phase function of the atmospheric particles. Not surprisingly, at such angles, $N$ should still be chosen high enough to resolve these angular features, otherwise the integration errors increase too much upon interpolation of the Fourier coefficients. Nevertheless, an interpolation method is worth being implemented, in particular for planetary atmospheres containing particles with rather smooth phase functions, and being explored further.

Finally, we will briefly discuss another optimization for the disk-integration method as described in this article. Namely, for a given value of $M$, the largest errors in the disk-integrated $a_{1}$ and $P_{\mathrm{s}}$ are generally due to sharp angular features in the singlescattering matrix of the particles in the planetary model atmosphere. These errors could be reduced without increasing $M$ by treating light that has been singly scattered within the planetary atmosphere separately from the multiple-scattered light, since the elements of the planetary scattering matrix of the multiplescattered light are much smoother functions of the scattering angle than those of the singly scattered light (see de Haan et al. 1987; Hovenier et al. 2004, for an explanation of how to separate singly from multiple-scattered light when using an addingdoubling method).

\section{References}

Chandrasekhar, S. 1950, Radiative transfer. (Oxford: Clarendon Press) Charbonneau, D., Allen, L. E., Megeath, S. T., et al. 2005, ApJ, 626, 523 Chauvin, G., Lagrange, A.-M., Dumas, C., et al. 2004, A\&A, 425, L29 Chauvin, G., Lagrange, A.-M., Zuckerman, B., et al. 2005, A\&A, 438, L29 de Haan, J. F., Bosma, P. B., \& Hovenier, J. W. 1987, A\&A, 183, 371 de Rooij, W. A. 1985, Reflection and Transmission of Polarized Light by Planetary Atmospheres (Ph.D. Thesis, Vrije Universiteit, Amsterdam) de Rooij, W. A., \& van der Stap, C. C. A. H. 1984, A\&A, 131, 237

Deming, D., Seager, S., Richardson, L. J., \& Harrington, J. 2005, Nature, 434, 740

Feldt, M., Turatto, M., Schmid, H. M., et al. 2003, in Earths: DARWIN/TPF and the Search for Extrasolar Terrestrial Planets, ESA SP-539, 99

Fridlund, C. V. M. 2004, Adv. Space Res., 34, 613

Gel'fand, I. M., Minlos, R. A., \& Shapiro, Z. Y. 1963, Representations of the Rotation and Lorentz Groups and their Applications (New York: Pergamon Press)

Hansen, J. E., \& Hovenier, J. W. 1974, in Exploration of the Planetary System, IAU Symp., 65, 197

Hansen, J. E., \& Travis, L. D. 1974, Space Sci. Rev., 16, 527

Horak, H. G. 1950, ApJ, 112, 445

Hough, J. H., \& Lucas, P. W. 2003, in Earths: DARWIN/TPF and the Search for Extrasolar Terrestrial Planets, ESA SP-539, 11

Hovenier, J. W. 1969, J. Atm. Sci., 26, 488

Hovenier, J. W. 1970, A\&A, 7, 86

Hovenier, J. W. 1971, A\&A, 13, 7

Hovenier, J. W., van der Mee, C., \& Domke, H. 2004, Transfer of Polarized Light in Planetary Atmospheres; Basic Concepts and Practical Methods (Dordrecht: Kluwer; Berlin: Springer)

Hovenier, J. W., \& van der Mee, C. V. M. 1983, A\&A, 128, 1

Kemp, J. C., Henson, G. D., Steiner, C. T., \& Powell, E. R. 1987, Nature, 326, 270

Saar, S. H., \& Seager, S. 2003, in ASP Conf. Ser., 529

Seager, S., Whitney, B. A., \& Sasselov, D. D. 2000, ApJ, 540, 504

Sromovsky, L. A. 2005, Icarus, 173, 284

Stam, D. M. 2003, in Earths: DARWIN/TPF and the Search for Extrasolar Terrestrial Planets, ESA SP-539, 615

Stam, D. M., De Haan, J. F., Hovenier, J. W., \& Aben, I. 2000, J. Geophys. Res., 22379

Stam, D. M., \& Hovenier, J. W. 2005, A\&A, 444, 275

Stam, D. M., Hovenier, J. W., \& Waters, L. B. F. M. 2004, A\&A, 428, 663

Stammes, P. 1992, in Symposium on Titan, ESA SP-338, 389

van de Hulst, H. C. 1957, Light Scattering by Small Particles (New York: J. Wiley and Sons)

van de Hulst, H. C. 1980, Multiple Light Scattering, Tables, Formulas, and Applications, Vols. 1 and 2 (New York: Academic Press)

Volten, H., Munoz, O., Hovenier, J. W., et al. 2005, J. Quant. Spectr. Rad. Trans., 90, 191 